\title{
Current approaches in lipid-based nanocarriers for oral drug delivery
}

\author{
María Plaza-Oliver $^{1,2} \cdot$ Manuel Jesús Santander-Ortega ${ }^{1,2} \cdot$ María Victoria. Lozano $^{1,2}$ (1)
}

Accepted: 13 January 2021 / Published online: 2 February 2021

(c) Controlled Release Society 2021

\begin{abstract}
Lipid-based nanocarriers have gained much interest as carriers of drugs with poor oral bioavailability because of their remarkable advantages like low toxicity, affordable scale-up manufacture, strong biocompatibility or high drug loading efficiency. The potential of these nanocarriers lies in their ability to improve the gastrointestinal stability, solubility and permeability of their cargo drugs. However, achieving efficient oral drug delivery through lipid-based nanocarriers is a challenging task, since they encounter multiple physicochemical barriers along the gastrointestinal tract, e.g. the gastric acidic content, the intestinal mucus layer or the enzymatic degradation, that they must surmount to reach their target. These limitations may be turned into opportunities through a rational design of lipid-based nanocarriers. For that purpose, this review focuses on the main challenges of the oral route indicating the strategies undertaken for lipid-based nanocarriers in order to overcome them. Understanding their shortcomings and identifying their strengths will determine the future clinical success of lipid-based nanocarriers.
\end{abstract}

Keywords Oral delivery $\cdot$ Liposome $\cdot$ Solid-lipid nanoparticle $\cdot$ Nanoemulsion $\cdot$ Nanocapsule $\cdot$ Self-emulsifying system

\section{Introduction}

Lipid-based nanocarriers are a landmark in drug delivery. Research on lipid-based nanocarriers has provided Doxil®, the first FDA-approved nanocarrier, and it is intensively progressing in areas like cancer or peptide delivery [1,2]. The great attention received by these nanocarriers in last decades is due, in part, to the excellent properties of their lipid components, such as high versatility, biocompatibility and low toxicity profile [3]. Lipid-based nanocarriers are suitable for enhancing the clinical potential of drugs with biopharmaceutical limitations, such as low aqueous solubility or stability, and for providing alternatives to the parental route. More specifically, the convenience and advantages of the oral route, e.g. ease of administration and high patient compliance, have strengthened the research on oral lipid-based nanocarriers. This is reflected

María Victoria. Lozano

mvictoria.lozano@uclm.es

1 Faculty of Pharmacy, Cellular Neurobiology and Molecular Chemistry of the Central Nervous System Group, 02008 Albacete, Spain

2 Regional Centre of Biomedical Research (CRIB), University of Castilla-La Mancha (UCLM), 02008 Albacete, Spain in the increasing number of patents in the field-promoting innovation and commercialisation [4-6]. An excellent example is found in the patent filed in 2006 by M. Kidron claiming methods and compositions based on lipids for the oral delivery of peptides [7]. This was the basis of Oramed, a lipid-based oral insulin delivery system that will soon initiate phase III clinical trials and perfectly shows the potential that lipid-based nanocarriers may have for efficient oral drug delivery [8].

The term lipid-based nanocarrier includes liposomes, self-nano and microemulsifying drug delivery systems, nanoemulsions and nanocapsules, among others (Fig. 1). All of them have in common that their formulation can be adapted to the characteristics and requirements of the oral route. Indeed, most oils and fats used for the development of these nanocarriers derive from dietary lipids facilitating oral permeability and biodegradability. Additionally, lipidbased nanocarriers can be designed to interact with specific cell populations in the gastrointestinal tract, increasing the drug delivery efficacy. However, oral drug delivery using lipid-based nanocarriers is a challenging task, since they must overcome the harsh gastrointestinal environment and multiple chemical and physical barriers. These barriers include the gastrointestinal fluids, which have variable and extreme $\mathrm{pH}$ values and contain salts and enzymes that 
Fig. 1 Lipid-based nanocarriers in oral drug delivery

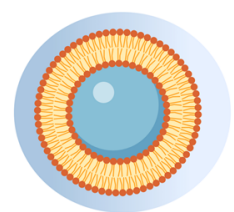

Liposomes

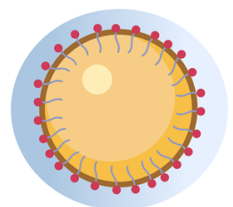

O/W nanoemulsions

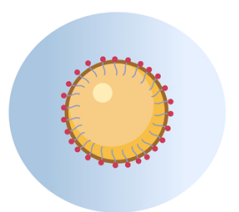

O/W microemulsions

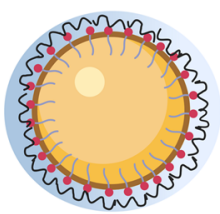

Nanocapsules

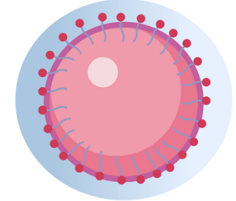

Solid lipid nanoparticles

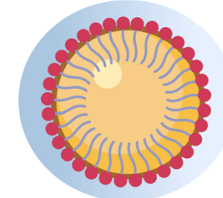

Self-nanoemulsifying systems

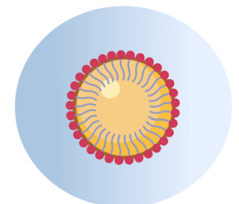

Self-microemulsifying systems

\section{Aqueous \\ phase

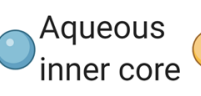 \\ Fluid lipid \\ inner core \\ Solid lipid \\ inner core \\ R S Surfactants $\begin{array}{r}3 \text { Polymer } \\ \text { coating }\end{array}$}

may compromise the stability of nanocarriers; a mucus layer colonised by the gut microbiota, which may limit the intestinal residence time and/or the access of nanocarriers to the intestinal epithelium; and the intestinal epithelium itself, which may express drug efflux mechanisms compromising the final absorption of the nanocarrier $[9,10]$.

These challenges may turn into opportunities if the current information about the gastrointestinal barriers and their interaction with nanocarriers is understood and used to develop strategies aimed to improve the oral delivery of lipid-based nanocarriers. The behaviour and performance of these nanocarriers in the gastrointestinal tract may be modulated through a rational selection of the lipid components and a design based on the drug properties and on the medical need. For instance, depending on their composition, lipid-based nanocarriers have the potential to avoid the degradation of gastric labile drugs, enhance their intestinal residence time through their adhesion to the mucus layer or inhibit intestinal drug efflux. On this context, this work aims to analyse the state-of-the art of the main approaches currently followed to enhance the oral delivery of drugs using lipid-based nanocarriers.

\section{Challenges for lipid-based nanocarriers in the oral route}

The gastrointestinal tract is a difficult environment for nanocarriers due to the aggressive conditions that they encounter. First, its wide $\mathrm{pH}$ gradient is a challenge to the physical and chemical stability of the nanocarriers. The more acidic conditions are found in the stomach $(\mathrm{pH} 1-2.5)$, whereas the colon reaches $\mathrm{pH} 7-8$ [9]. Most nanocarriers have ionisable groups on their surface, so $\mathrm{pH}$ values close to their isoelectric point would minimize or even remove their surface charge. Besides, gastrointestinal fluids include a variable concentration of salts and electrolytes, which may also negatively affect to the surface charge of nanocarriers. This can lead to destabilisation processes that may end with their aggregation in gastrointestinal fluids. Secondly, the gastric enzymes, like pepsin or gelatinase, may also compromise the stability of the nanocarriers. The enzymatic content is even higher in the duodenum, since it is rich in biliary and pancreatic secretions that include lipases, peptidases and amylases [9]. Taking into account the composition of lipid-based nanocarriers, it is reasonable to think that lipases may digest them leading to solubilisation, aggregation or precipitation [11]. Besides, cationic electrolytes such as $\mathrm{Ca}^{2+}$ or $\mathrm{Mg}^{2+}$ may form insoluble salts with some lipid components of the carriers, i.e. long-chain fatty acids, removing them from their and allowing their digestion by lipases [11]. Then, stability testing of lipidbased nanocarriers is essential in early development stages. To reproduce these conditions, researchers use simulated fluids that have $\mathrm{pH}$ values, electrolytes and enzyme concentrations similar to those of the gastric or intestinal fluids (USP XXIX) [12-14].

The next barrier that nanocarriers meet in the gastrointestinal tract is the mucus layer underlying the epithelium. Mucus is a complex hydrogel mainly composed of water and proteins. Mucins are the more abundant proteins in mucus; they entangle to form a viscoelastic gel that exhibits shear-thinning properties [15]. Most mucins in the intestinal mucus are glycosylated so it has negative 
charge. This may lead to the adhesion of positively charged nanocarriers to mucus through electrostatic interactions [15]. Mucus also includes additional carbohydrates, lipids, salts, cellular debris and antibodies. To elucidate the impact of mucus on oral drug delivery, mucus models of different complexity have been used. The ideal mucus model should recapitulate the characteristics and composition of human intestinal mucus as far as possible [16]. For this purpose, porcine intestinal mucus is widely used [17-20]. However, the properties of native mucus may vary with the animal species, age or disease state. In view of the high variability and complexity that entails the use of native excised mucus, other authors prefer to use purified mucin solutions [21, 22], which are easier to prepare and minimize variability of the results, although they do not mimic completely the mucus composition [16]. Intestinal mucus is structured in two different layers: a first layer, nearer to the intestinal lumen, which is loosely adhered, and a second layer, in contact to the epithelium, which is firmly adhered. The intestinal mucus has a protective function, impeding the contact of foreign particles with the epithelial cell, by the periodical renewal of the loosely adherent layer [15]. This can lead to a prompt clearance of nanocarriers from the intestinal tract, minimizing their opportunities to reach the epithelium. There are different in vitro and in vivo techniques to evaluate the interaction of nanocarriers with mucus, such as the mucin particle method, DLS, fluorescence recovery after photobleaching (FRAP) and particle tracking [12, 23]. Among these, particle tracking has gained special attention in the last years, due to the unique advantages it offers, such as providing individual and ensemble information about the mucodiffusion of particles in real time. For a deeper knowledge in current techniques for the determination of the mucoadhesion/diffusion in nanocarriers, authors are referred to exceptional reviews in the field [12, 23].

Mucus harbours a diverse and dynamic population of microorganisms, known as the gut microbiota, which offers a range of benefits to the host, such as protecting against pathogens, modulating the immune system or harvesting energy. The bacterial strains and their abundance vary along the gastrointestinal tract, being more numerous and diverse in the colon region. When microbial populations are altered, the condition is known as dysbiosis. This has been related to several diseases and pathological situations, such as intestinal bowel disease (IBD), carcinogenesis or obesity [24]. The importance of microbiota in the body homeostasis has led to an exponential research in the field [25]; despite of this, the knowledge about the interaction of microbiota with nanocarriers is still scarce [26]. It is important to consider that gut microbiota may have a relevant impact on oral administered nanocarriers, e.g. metabolism or retention, which could alter (positively or negatively) the final fate and efficacy of nanocarriers. Additionally, nanocarriers may influence the microbiota by exhibiting prebiotic or antibiotic-like behaviour [27].

The intestinal epitheliumis the last barrier for drugs in order to achieve the systemic circulation.Once associated to the nanocarrier, drugs can be released to the intestinallumen and absorbed by the intestinal cells or be taken up still encapsulated inthe nanocarrier. In this case, different mechanisms and cells are involved. Theintestinal epithelium is mainly constituted by enterocytes, although othercells like goblet cells, $\mathrm{M}$ cells, $\mathrm{L}$ cells or Paneth cells are also found [28].Enterocytes are polarized absorptive cells with a microvilli brush border intheir apical side, sealed among them by tight junctions. In general, themechanisms of uptake by enterocytes are paracellular, through the space betweencells, or transcellular, through enterocytes. The paracellular pathway plays aminor role in the absorption of drug and nanocarriers, since it only enablesthe transport of small and hydrophilic molecules. Opposite, hydrophobicnanocarriers/drugs may be transported by the transcellular pathway [9].The main transcellular pathways are endocytic and may occur by phagocytosis, via immune or M cells, or by pinocytosis, which can occur through differentmechanisms, such as macropinocytosis, clathrin- or caveolae-mediatedendocytosis and clathrin- and caveolaeindependent endocytosis [29].Then, the nanocarriers may follow different intracellular trafficking andtransformation pathways that may end with the nanocarriers and/or cargomolecule translocation across the intestinal epithelium to reach systemiccirculation. In other occasions, an intestinal retention of the nanocarrierand/or its cargo molecule may be desired, e.g. for locally treating intestinalalterations, such as IBD [30].The second and third most commonly found cells in the intestinal epithelium aregoblet and $\mathrm{M}$ cells, respectively. Goblet cells secret mucins that are part ofthe mucus layer, whereas $\mathrm{M}$ cells take up and sample antigens andmicroorganisms. M cells are found in the gut associated lymphoid tissue (GALT)or Peyer's patches, a region that is connected with the lymphatic system andthat is not covered by a thick mucus layer, in order to enable closerinteraction with antigens [28].Thus, $\mathrm{M}$ cells arise as an appealing entry point for lipid-based nanocarriers, as discussed in Sect.4.

In an attempt to simulate the complex human intestinal environment, models of different complexity have been developed to predict the biological behaviour and the therapeutic potential of oral nanocarriers. Caco-2 cells, derived from human colorectal adenocarcinoma, have been widely exploited for modelling the intestinal barrier, since they can be polarized and differentiated as human enterocytes [31]. Additional in vitro models have been proposed to achieve a closer intestinal resemble, such as the co-culture of Caco-2 cells with HTM-29 MTX cells (mucus secreting model) [32] and/or with Raji cells (FAE model) [33-35]. Although these models are 


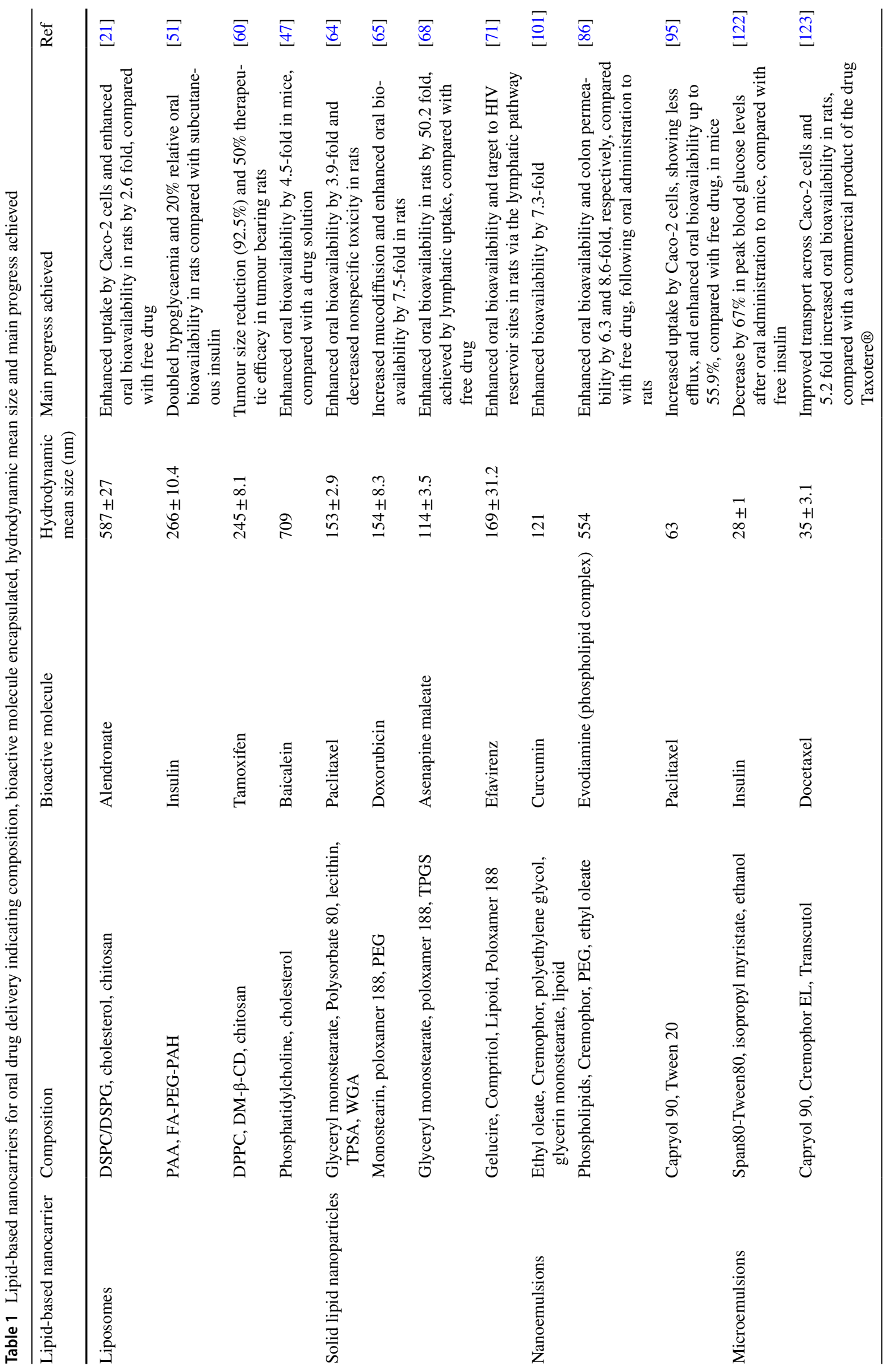




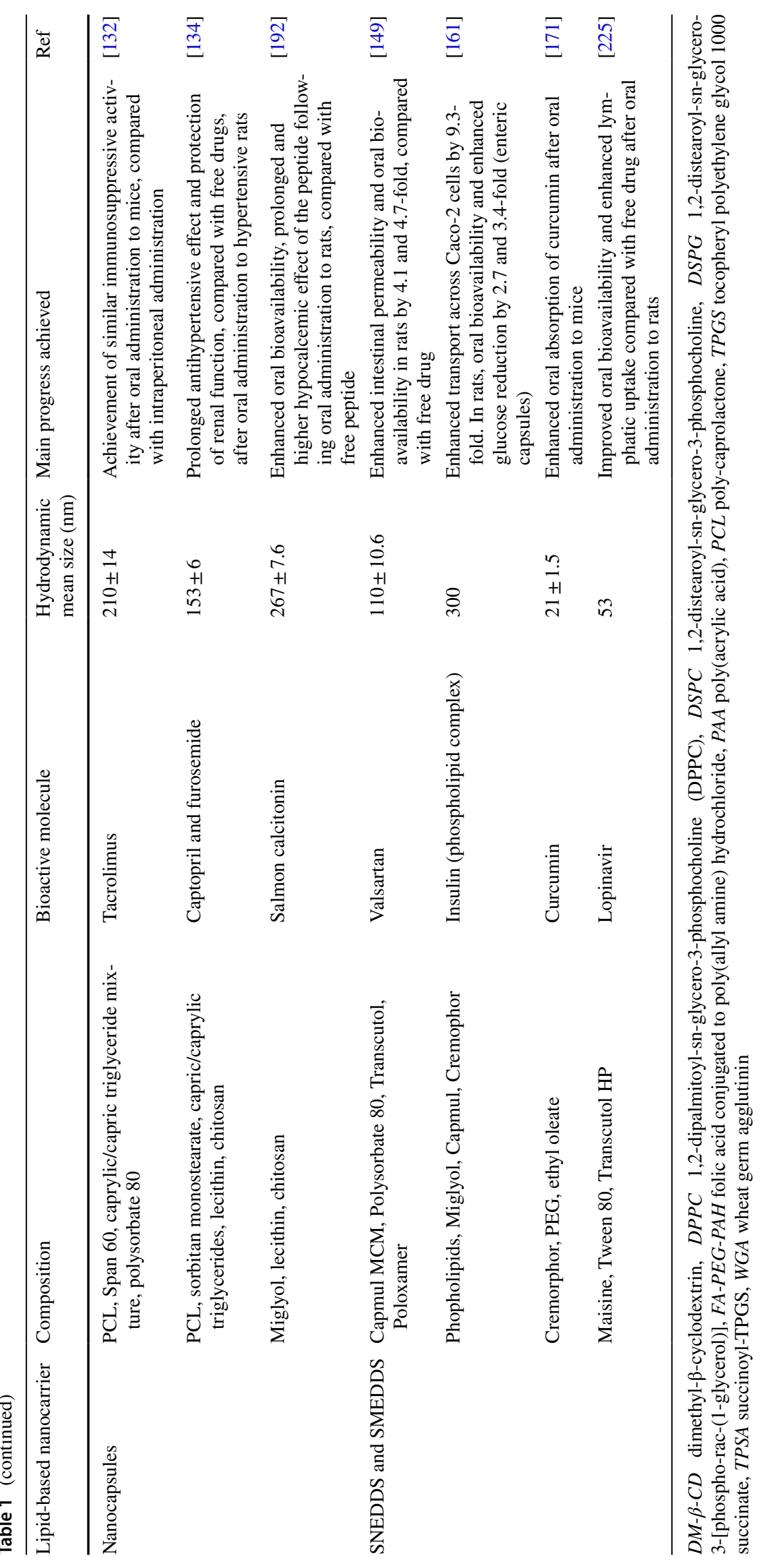


well-established, they show some limitations, e.g. they fail to mimic the crypt intestinal structure and they cannot include intestinal microbiota. Within this scenario, different 3D intestinal models have been developed in the last years by microfabrication (engineered intestinal tissues) or microfluidics (gut-on-a-chip). The engineered intestinal tissues reproduce the intestinal 3D structure by using scaffolds composed of biomimetic materials, e.g. collagen [36], whereas gut-on-a-chip devices are able to mimic the intestinal biophysics forces and may allow the inclusion of microbiota strains and vascular components [37, 38].

The development of nanocarriers normally includes testing them in vivo in more advanced research stages. In this regard, the animal model, age and gender should be selected depending on the medical need and the specific aim of the study [39]. Rats and mice are the most commonly used species for the in vivo testing of oral nanocarriers, since their gastrointestinal tract resembles that of the humans, although they also show some peculiarities, e.g. different gut microbiota to humans and absence of gall bladder, which may require consideration. On the other hand, for toxicological or biopharmaceutical studies, animal model with a similar human metabolic pattern of that from humans is desirable [39]. Additionally, disease models may be used for understanding the therapeutic potential of nanocarriers, as well as their biological performance (e.g. absorption) in the pathological state. A more detailed vision of in vitro and in vivo intestinal models is beyond the scope of this work, but it is excellently reviewed elsewhere [12, 39-41].

\section{Oral lipid-based nanocarriers}

The diverse components and preparation methods currently available have led to a wide variety of lipid-based nanocarriers with the potential to enhance drug oral bioavailability, as depicted in Table 1. Although their specific composition determines their properties and biological performance, all these nanocarriers have in common the use of lipid ingredients, such as vegetal oils, free fatty acids or liposoluble vitamins. The following subsections specify the main characteristics and achievements of different lipid-based nanocarriers.

\section{Liposomes}

Liposomes are spherical vesicles constituted by lipid bilayers and an aqueous inner core. Conventional liposomes are made of phospholipids and sterols, such as cholesterol, which are usually incorporated in the formulation to stabilize the liposomal membrane [42, 43]. These nanocarriers cover sizes from $10 \mathrm{~nm}$ up to several microns. Liposomes were first described in the early 1960 s
[44], opening the door to intense and fruitful decades of investigation in the drug delivery field $[45,46]$. Since then, several liposome-based formulations intended for parental administration have been approved for clinical use in humans by the FDA.

The promising antecedents provided by injectable liposomes, as well as their biocompatibility, have encouraged the development of liposomes as carriers of drugs with poor bioavailability by the oral route. The structure and composition similarities of liposomes with cell membranes may contribute to the permeation enhancement across the intestinal epithelium. Besides, to overcome the physicochemical barriers that they may encounter and to modulate their behaviour in the gastrointestinal tract, the surface of liposomes can be modified, e.g. by the addition of cationic surfactants, bile salts or targeting ligands [43].

Liposomes can be spontaneously formed by hydration of phospholipids. The traditional method for the formulation of liposomes is the film hydration method. In this method, phospholipids are dissolved into organic solvents that are later evaporated, obtaining a phospholipid film that is hydrated to yield an aqueous dispersion of liposomes. Other conventional approach followed to prepare liposomes is the reverse-phase evaporation technique. In this method, the phospholipid solution in organic solvents is mixed with the aqueous phase, and the organic solvents are further evaporated to obtain the liposomes [42].

Thanks to their composition, liposomes allow the encapsulation of both hydrophilic and hydrophobic drugs. Hydrophilic molecules can be loaded into their inner cavity, whereas hydrophobic drugs can be included into the lipid bilayer. Regarding hydrophilic molecules, liposomes have shown promise to increase the oral bioavailability of baicalein [47] and it is worth to highlight their growing contribution in the oral delivery of peptides, including insulin or lactoferrin [2,48-54]. Lactoferrin is a peptide that has attracted much interest due to its important role in the immune system, showing antioxidant, antiinflammatory, antibacterial and antiviral activity. However, it is susceptible to hydrolysis by gastrointestinal enzymes, what hampers its oral bioavailability. The encapsulation of lactoferrin in liposomes has been researched as a strategy to overcome this limitation. Indeed, there is a current clinical trial in phases 2 and 3 that study the efficacy and safety of lactoferrin-loaded liposomes orally administered in COVID-19 patients with mild to moderate disease and asymptomatic patients (NCT04475120) [55]. This is an example of thepotential that liposomes may have for current oral drug delivery challenges. With concern to hydrophobic molecules, liposomes have been exploited to improvethe oral bioavailability 
of drugs such as cyclosporine A [56]or fenofibrate [57], among others. Liposomes are also promising for the achievement of efficient andsafe oral cancer treatments [58-60], as recently reported by Ağardan et al. These authorsdescribed that liposomes loaded with tamoxifen and dimethyl- $\beta$-cyclodextrin aspermeation enhancer are able to achieve a $92.5 \%$ reduction of the tumour areaafter their oral administration to tumour bearing rats, without a significantweight loss [60].

\section{Solid lipid nanoparticles}

Solid lipid nanoparticles are nanocarriers composed by a lipid core with a monolayer surfactant shell that stabilize them to form aqueous dispersions at the nanometer range. Commonly used lipids for the production of solid lipid nanoparticles include triglycerides, fatty acids, waxes or phospholipids, whereas poloxamers, lecithin, polysorbates and bile salt derivatives are used as surfactants [61]. Additionally, surface modification of solid lipid nanoparticles might provide them with mucoadhesion properties. These nanocarriers started to gain attention in the early 2000s due to their advantages, such as their biodegradability and biocompatibility, as well as preparation possibilities without needing organic solvents.

The main peculiarity of these nanoparticles is that they remain solid both at room and body temperatures [61, 62]; this may increase their stability compared with liquid nanocarriers. Nevertheless, the solid state can also be a disadvantage of these nanocarriers, since the crystalline state of the solid may limit their drug-loading capacity. Moreover, encapsulated drugs may be promptly released from the nanoparticles in case of crystallisation during storage. However, lipid crystallisation might be retarded by the small size of the nanoparticles and the presence of surfactants, and further monitored by using techniques such as differential scanning microscopy or X-ray diffractometry [61]. Different preparation methods have been described for the production of solid lipid nanoparticles, including high pressure homogenisation, solvent emulsification/ evaporation, solvent injection and microemulsion method.

Solid lipid nanoparticles enable the delivery of both hydrophobic and hydrophilic molecules, so they have shown great potential to enhance the oral bioavailability of a wide variety of molecules, including anti-tumour drugs, e.g. paclitaxel or docetaxel [63-66], central nervous system drugs, e.g. lurasidone or asenapine [67, 68], cardiovascular drugs, e.g. carvedilol or cilnidipine [69, 70], or antiviral drugs [71]. As an example, Dudhipala et al. reported that solid lipid nanoparticles loaded with rosuvastatin calcium increase the bioavailability of the drug following oral administration to rats, compared with a rosuvastatin calcium suspension by more than 4 times. Besides, authors reported a decrease in lipid profile in hyperlipidemic rats for $36 \mathrm{~h}$, which was at least extended by $12 \mathrm{~h}$ in comparison with that of the suspension. This could reduce the dosing frequency of the treatment [72]. Similarly, solid lipid nanoparticles enabled to improve both pharmacokinetic and pharmacodynamics profiles of cilnidipine after oral administration to rats, compared with free drug. Concretely, oral area under the curve (AUC) was 2.4-fold increased and a $38 \%$ decrease in systolic blood pressure was achieved, maintaining more than a $20 \%$ decrease for $64 \mathrm{~h}$, compared with free cilnidipine [69].

\section{Nanoemulsions}

Nanoemulsions are dispersions of an oily and an aqueous phase stabilised by an appropriate surfactant or combination of surfactants with size values over $100 \mathrm{~nm}$ [73-77]. Nanoemulsions are thermodynamically unstable, what may lead to destabilisation processes such as coalescence or flocculation. However, the kinetics of the destabilisation is normally slow, i.e. weeks or months, so they can be considered as kinetically stable [75, 78]. Biphasic nanoemulsions can be oil in water $(\mathrm{o} / \mathrm{w})$ nanoemulsions, in which oily droplets are dispersed in the aqueous phase $[17,18,30,79-85]$ and water in oil (w/o) nanoemulsions, in which aqueous droplets are dispersed in the oily phase $[86,87]$. Multiple nanoemulsions can also be generated by mixing pre-formulated nanoemulsions with a second aqueous or oily phase. The more studied multiple nanoemulsions are w/o/w, which can be obtained by dispersing w/o nanoemulsions in an aqueous phase.

The different preparation methods to obtain nanoemulsions can be classified into high-energy or low-energy approaches. High-energy methods, such as ultrasonic homogenisation or microfluidisation, usually entail high formulation temperatures, what may limit their use of thermo labile molecules like peptides [73, 74, 77, 88-94]. Low-energy methods, including solvent displacement technique and phase inversion, are performed in milder conditions [74]. Indeed, solvent displacement technique can be used to obtain nanoemulsions loaded with peptides, since it avoids high temperatures [2].

In last decade, the great versatility of nanoemulsions has enabled to exploit their potential as oral carriers of anti-tumour drugs [89, 93, 95-97]. In this line, Pangeni et al. developed multiple nanoemulsions loaded with 5-fluorouracil and a deoxycholic acid derivative of oxaliplatin. The permeability of both anti-tumour drugs across differentiated Caco-2 cells was enhanced, and their oral bioavailability in rats was 1.39and 9.19-fold higher than free oxaliplatin and 5-fluoruracil, respectively. Authors also reported a tumour growth reduction 
of $73.9 \%$ in CT26 tumour-bearing mice when they were orally treated with the loaded nanoemulsion, compared with the control group [97]. Choi et al. followed a similar approach, developing multiple nanoemulsions loaded with a deoxycholic acid derivative complex of oxaliplatin (OXA/DCK-NE), whose structure is depicted in Fig. 2a. The system showed a 3.35- and 1.73-fold increase in the complex (OXA/DCK) permeability through differentiated Caco-2 cells and oral bioavailability in rats, respectively. Furthermore, the oral administration of the nanoemulsion comprising oxaliplatin complex impeded tumour growth by $>60 \%$ in B16F10.OVA tumour-bearing mice. This tumour inhibition was further increased to $\sim 78 \%$ when OXA/ DCK-NE was combined with an anti-programmed cell death protein-1 ( $\alpha$ PD-1) antibody, as shown by Fig. 2b, c [98].

a

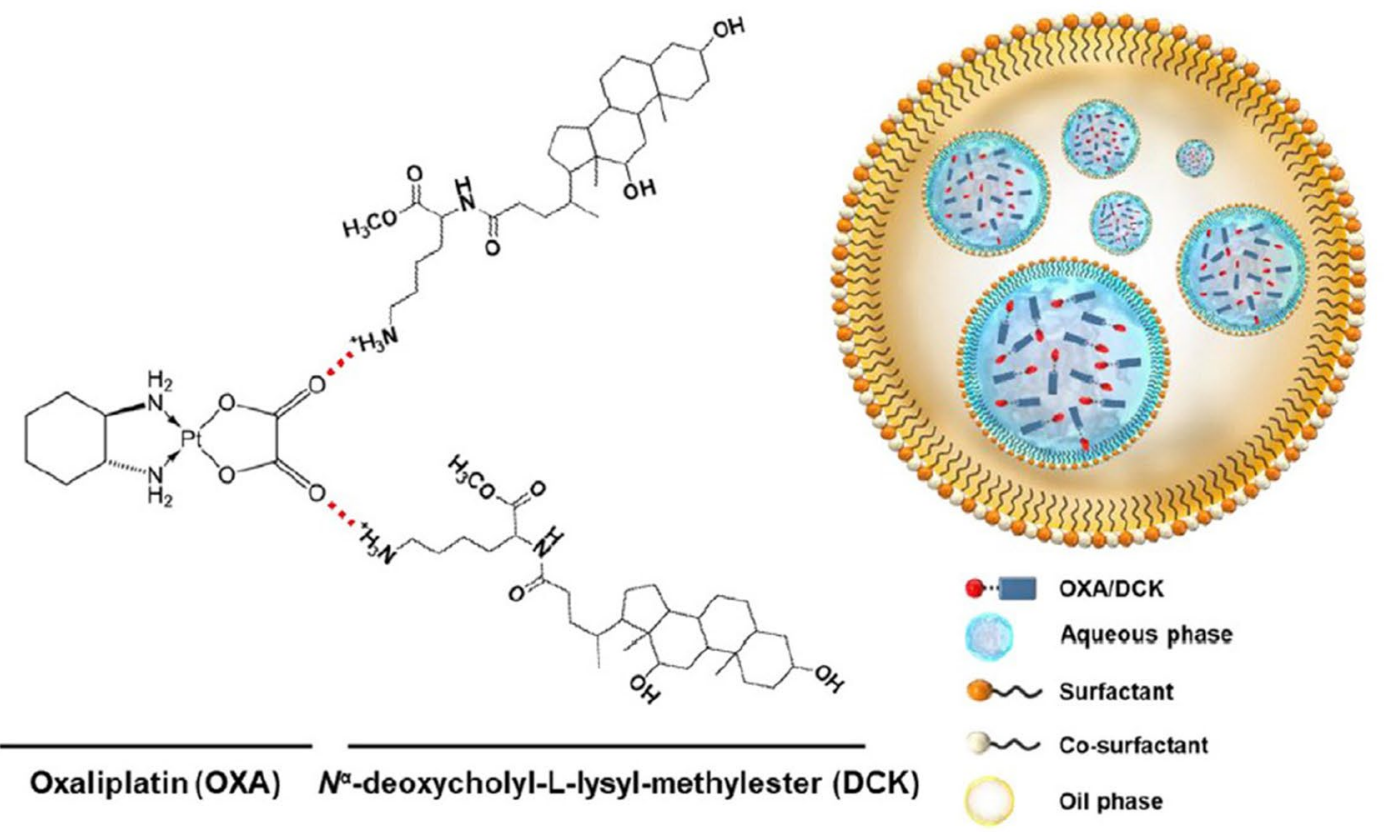

b

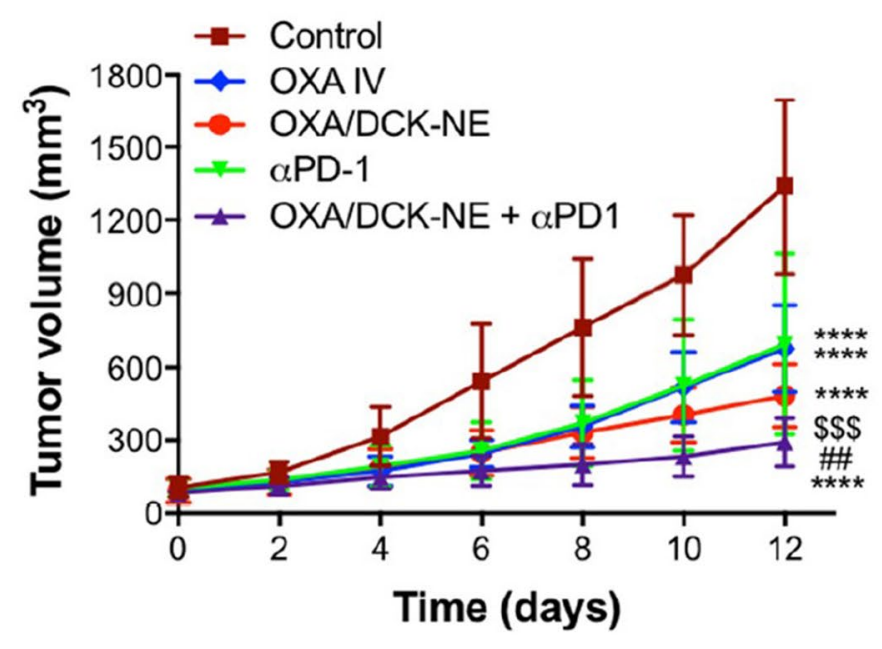

C

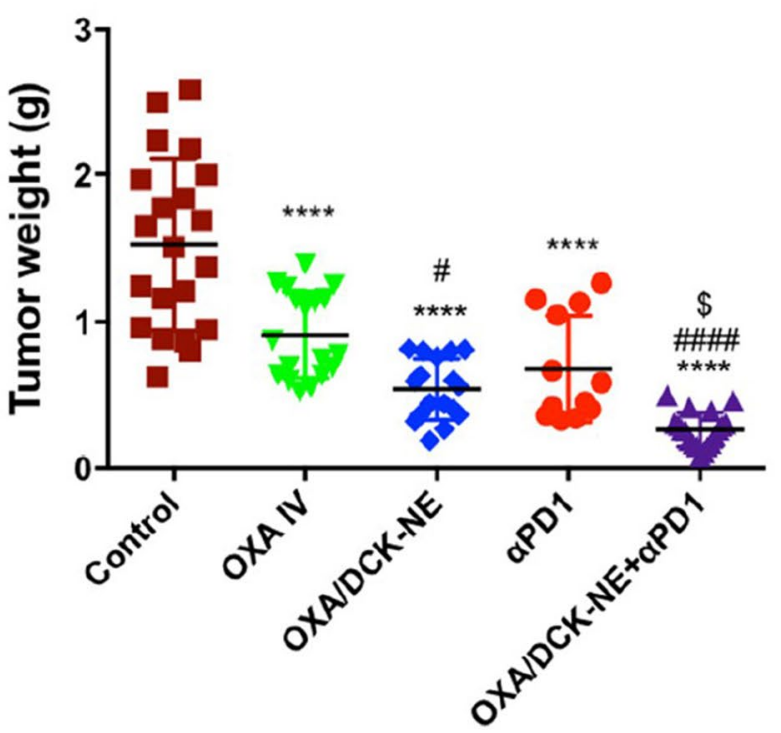

of tumour tissue in each group ( $n=18$ for each group). Data are presented as mean \pm standard error of the mean. ${ }^{* * * *} p<0.0001$ compared with the control. \#p $<0.05, \# p<0.01, \# \#$ \# $<0.0001$ compared with OXA IV. $\$ p<0.05, \$ \$ \$ p<0.001$ compared with $\alpha \mathrm{PD}-1$. OXA: oxaliplatin; DCK: $\mathrm{N} \alpha$-deoxycholyl-L-lysyl-methylester (bile salt derivative); OXA/DCKNE: OXA/DCK nanoemulsion; OXA IV: intravenous oxaliplatin; $\alpha$ PD-1: $\alpha \mathrm{PD}-1$ antibody. Adapted with permission of [98] 
The promising potential of nanoemulsions in oral drug delivery shown by these works has been also reported for other drugs such as saquinavir [81], dabigatran [99], granisetron [83] or anti-hypertensive drugs, e.g. candesartan or olmesartan [82, 100]. Besides, the interest of nanoemulsions for improving the oral delivery of nutraceuticals and natural extracts and molecules with therapeutic potential, e.g. curcumin, quercetin, flavones or carotenoids derivatives, has also been increased [30, 84, $85,101-110]$. In fact, the ability of orally administered curcumin-loaded nanoemulsion to treat obese women with high risk of breast cancer is being assessed in a clinical trial (NCT01975363) [111]. A second trial is currently recruiting participants to evaluate the potential of curcumin oral nanoemulsions to reduce joint pain in breast cancer survivors with aromatase inhibitor-induced joint disease (NCT03865992) [112].

\section{Microemulsions}

Microemulsions are colloidal dispersions of two immiscible phases stabilized by surfactants added in higher concentrations $(\geq 20 \%)$ than the required to obtain nanoemulsions (3-10\%). This confers microemulsions with thermodynamic stability $[75,113]$.

Microemulsions usually exhibit mean sizes lower than nanoemulsions [75]. However, there is no established size threshold that finely separates nanoemulsions from microemulsions, although microemulsions usually have size values below $100 \mathrm{~nm}$ [114-116]. Another distinctive characteristic of microemulsions is their capacity to adopt different shapes, apart from spherical, e.g. hexagonal or worm-like, what could have implications on the flow characteristics of the systems. This could affect their biodistribution or diffusion through biological barriers, as observed for other type of differently shaped nanocarriers $[117,118]$. Besides, although not deeply studied, it has been proposed that the interaction of nanocarriers with cellular membranes and/or their endocytosis may also be influenced by their shape $[119,120]$.

Regarding their preparation methods, microemulsions are spontaneously formed by mixing all their components. This is due to the low interfacial tension provided by the high surfactant concentration. This has been proposed as a practical method to differentiate microemulsions from nanoemulsions, since nanoemulsions are only obtained after adding the surfactant to the oil before mixing the oily phase to the aqueous phase [78].

During last the years, microemulsions have been developed to enhance the oral bioavailability of drugs such as heparin, insulin or docetaxel [121-123]. In this sense, low-molecular weight heparin was complexed with deoxycholic acid and incorporated into microemulsions of tricaprylin as the oily phase and a mixture of Tweens 80 and 20 as surfactants. The administration of the microemulsion significantly enhanced the oral bioavailability of the conjugate to $1.5 \%$ in mice without causing intestinal damage [121]. More recently, Li et al. have proposed w/o microemulsions loaded with insulin as oral delivery systems of the peptide. These authors observed a significant decrease of $67 \%$ in peak blood glucose levels in response to the glucose tolerance test when the microemulsion was orally administered to mice [122]. Microemulsions have also shown potential for the oral delivery of nutraceuticals, like curcumin, lycopene or myricetin [107, 114, 115]. In this line, Guo et al. have recently developed a lycopeneloaded microemulsion based on limonene as oil, Tween 80 as surfactant and Transcutol HP as co-surfactant. The microemulsion, sized $<15 \mathrm{~nm}$, doubled the lycopene oral bioavailability in rats compared with a control oily solution of lycopene. Interestingly, authors found that lycopene microemulsion showed a preferable distribution in brain after its oral administration to mice, showing promise as an oral drug delivery system targeting the brain [114].

\section{Nanocapsules}

Lipid nanocapsules are vesicular nanocarriers constituted by an oily phase and an aqueous phase, stabilised by surfactants and a polymeric shell. Many physicochemical properties, and therefore the biological identity, of nanocarriers depend on their surface characteristics. Thus, the polymeric shell of nanocapsules has a relevant impact on their properties, e.g. surface charge or hydrophilicity, and gastrointestinal behaviour, e.g. interaction with mucus and/or enzymes. Some polymers widely used in the preparation of lipid nanocapsules include polyethylene glycol (PEG) derivatives, chitosan, hyaluronic acid, caprolactone, Eudragit or PLA.

The preparation of nanocapsules involves two steps: first, the formulation of nanoemulsions and second, the formation of the polymeric shell, which can be achieved through a variety of methods, including interface polymerisation [124, 125] or interfacial deposition [126], among others.

Nanocapsules have been applied to enhance the oral bioavailability of a wide variety of drugs, including fondaparinux [127], sn-38 [128], paclitaxel [129, 130], exenatide [131], tacrolimus [132], psorialidin [133] or furosemide [134], as well as of nutraceuticals, such as curcumin [135], lutein [136] or resveratrol [137, 138]. As an example, Peltier et al. developed paclitaxel-loaded nanocapsules that were able to enhance the bioavailability of the anti-tumour drug after oral administration to rats. Concretely, the AUC of paclitaxel was increased about 
threefold, compared with free paclitaxel [130]. More recently, Michalowski et al. have developed multiwall chitosan lipid nanocapsules loaded with furosemide that included a captopril-functionalised shell. The nanocapsules sized $<200 \mathrm{~nm}$ and had positive charge. After their oral administration to spontaneously hypertensive adult rats, authors observed a prolonged antihypertensive effect compared with control solutions of captopril and furosemide. Besides, they reported a potential renal protective role of the loaded functionalised nanocapsules, which showed great promise as an innovative antihypertensive treatment [134].

\section{Self-nano and microemulsifying drug delivery systems}

Although self-emulsifying drug delivery systems, also known as emulsion preconcentrates, were first described by Panton et al. in 1985 [139], this type of formulation has experienced massive grown in the last 15 years, as shown in Fig. 3. Self-nanoemulsifying drug delivery systems (SNEDDS) are anhydrous forms of nanoemulsions. They offer several advantages over conventional nanoemulsions, based on their composition. First, as they do not include water, they show better long-term stability profiles and allow the administration of lower volumes. Additionally, they can be incorporated within dosage vehicles such as soft/ hard gelatin or hydroxypropyl methylcellulose capsules, what improves their palatability and therefore the patient compliance [113, 140, 141].

SNEDDS are mixtures of drug, oil and surfactant. If required, cosurfactant or solubilisers may be added. The main characteristic of SNEDDS is that they spontaneously form nanoemulsions when introduced in an aqueous environment under mild stirring, e.g. the gastrointestinal

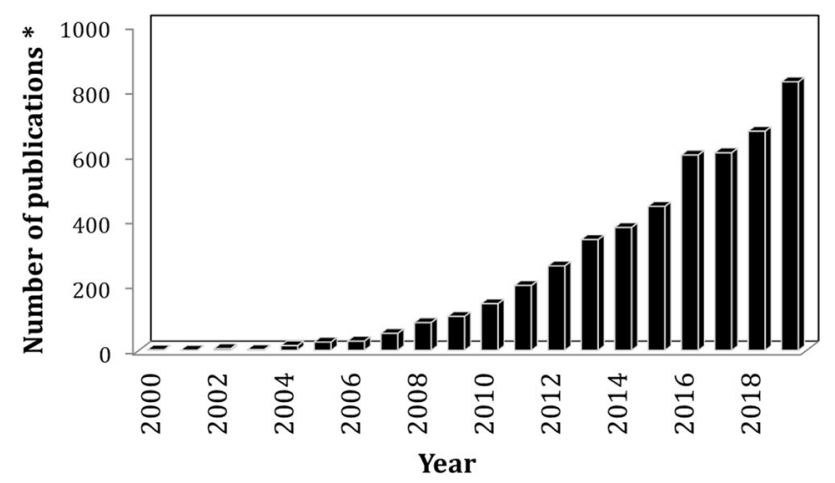

Fig. 3 Publication tendency of scientific papers focused on SNEDDs and SMEDDs in the last 20 years. Data obtained from the Scopus database, filtering publications that included the words "self-nanoemulsifying drug delivery system", "SNEDD", "self-microemulsifying drug delivery system" and/or "SMEDD" motility [84, 113, 114, 114, 115]. According to Reiss et al., the free energy required for self-emulsification depends on the number and radius of the dispersed droplets as well as on the interfacial tension of the system [142]. The high surfactant ratio of SNEDDS allows the reduction of the energy threshold required for the dispersion of oil droplets up to zero or negative values and so the system tends to emulsify [140, 143]. Similarly, self-microemulsifying drug delivery systems (SMEDDS) turn to microemulsions in aqueous media.

Self-emulsifying systems have been developed in last years to increase the oral bioavailability of antiviral [144, 145], cardiovascular [146-150], anti-tumour [151-158], antiemetic [159] or immunosuppressive [160] drugs, as well as of peptides [161-165], vitamins [166], nutraceuticals and natural compounds, such as resveratrol, curcumin or bruceine [167-173].

Despite the apparent simplicity of SNEDDS and SMEDDS preparation, their design must be carefully performed. First, their oil core should solubilize the maximum possible amount of drug and to enable the emulsification of the system in fine droplets once in contact with an aqueous phase. In order to achieve this double objective, a mixture of oils may be required [146]. Secondly, the gastrointestinal fluids that constitute the aqueous phase of SNEDDS and SMEDDS should also be considered during their development, since temperature, electrolytes or $\mathrm{pH}$ variations could affect the stability of the system and their emulsification [113]. Simulated gastric and intestinal fluids can be used to predict the emulsifying properties of the system, e.g. emulsifying time. In this line, it is important to consider that gastrointestinal alterations, such as IBD or bacterial infections, may alter the intestinal conditions, what should be considered during the design and development of oral self-emulsifying drug delivery systems for these diseases.

Besides, conventional SNEDDS and SMEDDS may present serious limitations, such as the long-term incompatibility of their components with capsule shells or drug precipitation during low-temperature storage. To minimize these problems, current efforts are being made to obtain solid SNEDDS and SMEDDS, which are more stable and convenient for longterm storage. In this line, Taha et al. developed oral solid tablets based on vitamin A SNEDDS, composed by soybean oil, Cremophor and Capmul. Tablets were obtained by adsorption of the formulation to microcrystalline cellulose and compression of the powder. These authors observed that the oral bioavailability of vitamin A was enhanced by 1.4 -fold, showing higher peak plasma concentration (Cmax) and a rapid onset of action (faster Tmax) compared with an oily solution of vitamin A when administered to rats [166].

As a result of the intense research of last decades focused on this type of lipid-based nanocarriers, several oral SNEDDS and SMEDDS have been marketed. A well-known example is Neoral cyclosporine formulation (Novartis), which was 
approved by the FDA in 1995. It includes corn oil mono, di and triglycerides and polyoxyl 40 hydrogenated castor oil as surfactant. This formulation is able to spontaneously form a system of less than $100 \mathrm{~nm}$ when dispersed in an aqueous media [174]. Another example of marketed self-emulsifying system is Norvir (Abbott), which was designed to increase the oral bioavailability of the anti-HIV drug ritonavir and it is currently used in clinics [175]. The clinical translation of these self-emulsifying systems has encouraged the development of novel nanocarriers able to improve the oral delivery of anti-HIV drugs. In this line, Lei et al. proposed a novel ritonavir-loaded SMEDDS that exhibited improved bioavailability and reduced gastrointestinal side effects, compared with Norvir [176].

\section{Strategies for oral delivery via lipid-based nanocarriers}

Different strategies have been proposed to enhance the potential of oral lipid-based nanocarriers by overcoming the physical and chemical barriers that they may encounter along the gastrointestinal tract. This includes the modulation of the interaction between nanocarriers and mucus, the targeting to specific cell populations or uptake pathways (i.e. lymphatic transport) or the inhibition of intestinal drug efflux (Fig. 4). It is worth to remark that the design of oral nanocarriers should involve not a single but a combination of absorption-enhancing strategies, e.g. diffusion through

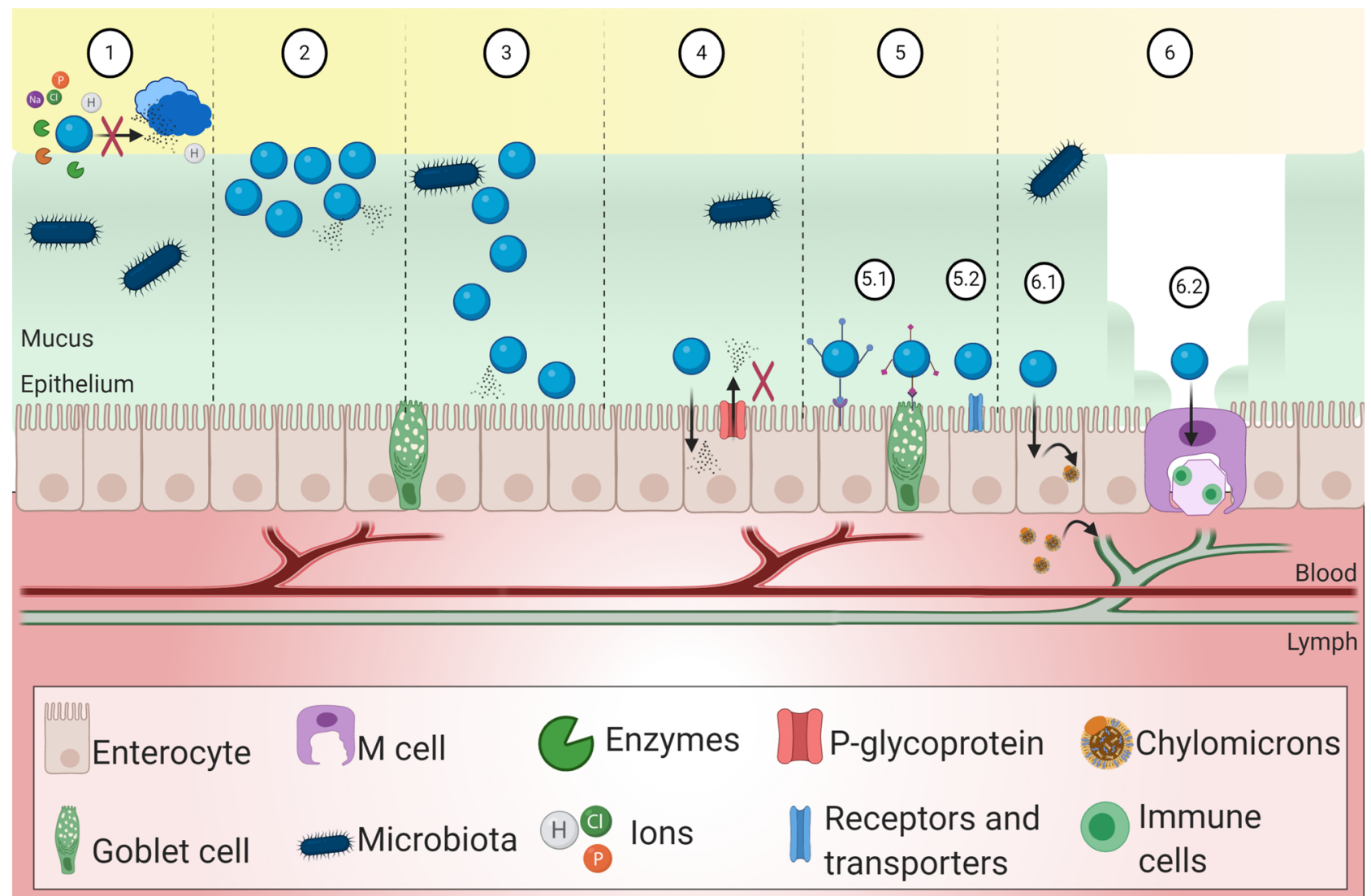

Fig. 4 Strategies for efficient oral delivery via lipid-based nanocarriers. (1) Improvement of the stability of nanocarriers in the harsh gastrointestinal environment that includes enzymes, salts and microbiota. (2) Enhancement of mucoadhesion. Nanocarriers remain adhered to the mucus and thereby their residence time is increased. The cargo molecule may be released. (3) Enhancement of mucodiffusion. Nanocarriers diffuse through the mucus, increasing the chances for their interaction with the epithelium. (4) Inhibition of P-glycoprotein. Drug efflux may be decreased, increasing drug effective absorption.
(5) Active targeting. (5.1) The surface of lipid-based nanocarriers can be functionalised with ligand that interact with specific cell populations, e.g. enterocytes or goblet cells. (5.2) Lipid-based nanocarriers can act as targeting ligands by themselves. (6) Enhancement of lymphatic transport, transport pathway that avoids first pass effect. (6.1) Chylomicrons, including lipids and hydrophobic cargo molecules from the internalised nanocarriers, are generated within enterocytes and absorbed by the lymphatic system. (6.2) Lymphatic uptake can be also achieved via $\mathrm{M}$ cells 
mucus and inhibition of drug efflux. This section reviews the recent advances on oral lipid-based nanocarriers, and the strategies followed.

\section{Improvement of the gastrointestinal stability}

The gastrointestinal tract has a complex medium in which different species, such as electrolytes, can reduce the surface charge of lipid-based nanocarriers. According to the DLVO theory, the interaction between two approaching nanoparticles can be expressed as the balance between an attractive potential of interaction (caused by the hydrophobic Van der Waals forces) and a repulsive electrostatic potential of interaction (caused by the ionisable groups of the nanoparticle). In this respect, the loss of electrostatic repulsive forces may lead to the destabilisation and aggregation of the nanocarriers [12, 177, 178]. Besides, the presence of bile salts and enzymes, such as lipases or phospholipases, may also affect their stability, compromising their integrity. The instability of nanocarriers may lead to the prompt leakage of their cargo drugs, what could negatively affect their oral bioavailability. The stability of lipid-based nanocarriers depends on their composition. For example, the polymeric shell of nanocapsules or the solid core of solid lipid nanoparticles may protect them from enzymatic degradation [2]. Regarding the lipid matrix, long-chain fatty acids are associated to slower degradation rates, compared with medium- and short-chain fatty acids $[179,180]$.

A strategy commonly followed to enhance the gastrointestinal stability of lipid-based nanocarriers is to modify their surface with hydrophilic polymers, such as PEG. On one side, the steric stabilisation granted by polymer chains may compensate electrostatic instability. On the other side, the hydrophilic surface may provide further stability by the enhancement of hydration forces [12]. It can also help to avoid the adsorption of enzymes and other surface active molecules to the nanocarriers. In this sense, Kashanian et al. demonstrated that the coating of solid lipid nanoparticles with PEG-stearate entailed higher stability in gastric simulated fluids, compared with uncoated nanoparticles [181]. Coating lipid-based nanocarriers with chitosan may also have a positive effect on their stability in gastrointestinal media, as observed by Garcia-Fuentes et al. These authors reported that chitosan-coated tripalmitin solid lipid nanoparticles remained practically unaltered in gastrointestinal simulated fluids [182]. Both the molecular weight and the acetylation degree affect to the stability of nanocarriers offered by chitosan. Concretely, low molecular weight chitosan with acetylation degree around 50\% was proposed as optimal stabilisation conditions for the nanocapsules. This was probably due to the achievement of a more hydrophilic surface, what enabled the stabilisation of nanocapsules through hydration forces, compared with chitosan with higher molecular weight and lower acetylation degree [183].

Bile salts have also been proposed as stabilizing agents against enzymatic degradation. For example, Niu et al. demonstrated that insulin-loaded liposomes containing sodium glycocholate provided increased protection against gastrointestinal enzymatic degradation, compared with conventional liposomes [184]. Similar results were reported in a separated work, which concluded that insulin-loaded liposomes containing sodium glycocholate showed no significant size variations and were able to retain more entrapped insulin, in comparison with conventional liposomes [185].

Traditionally, enzymatic degradation of lipid-based nanocarriers has been considered as an issue to avoid or at least minimize. Nevertheless, it is important to highlight that under certain circumstances, where drug integrity is not a concern, enzymatic degradation may lead to the solubilisation of the carriers and the formation of mixed micelles, which will be easily absorbed by enterocytes [186, 187]. Indeed, it is believed that the composition of lipid-based nanocarriers may determine the type and degree of interaction with gastrointestinal enzymes, and thereby the negative (e.g. precipitation) or positive (e.g. solubilisation) effects on the oral bioavailability of their cargo payloads. Additionally, the adsorption of enzymes to the surface of the nanocarriers could lead to the formation of an enzymatic corona that may affect the mucodiffusion behaviour of the nanocarriers.

\section{Interaction with the intestinal mucus}

The intestinal mucus is a strong barrier for the penetration of nanocarriers through the intestinal mucosa. A strategy that can be used to overcome this obstacle is to directly disrupt the mucus layer by the use of mucolytic excipients, such as $\mathrm{N}$-acetyl cysteine or papain $[188,189]$; however, this could lead to undesired effects on the physiology of the mucosa $[15,190]$. Mucus renewal, based on the elimination of the loosely adhered layer, has a protective function against pathogens, but it may also entail a prompt clearance of oral nanocarriers. Thus, many efforts have been driven towards the development of adhesive nanocarriers that are able to remain adhered to mucus. The aim is to increase the residence time of nanocarriers in the gastrointestinal tract and their possibilities to interact with the epithelium to promote drug absorption. Another approach is to develop nanocarriers that minimize the interactions with the mucus in order to diffuse across it before clearance and renewal occur [191]. These two complementary approaches have been recently merged by researchers to balance the mucoadhesion/mucodiffusion properties of nanocarriers 
in order to enhance their chances to reach the intestinal epithelium.

In this sense, lipid-based nanocarriers offer unique advantages. On one side, they enable the encapsulation of hydrophobic molecules in their oily cores, what may enhance the gastrointestinal stability and permeability of their cargo molecules across the intestinal epithelium, as previously discussed. On the other side, their surface can be easily modified with a wide variety of polymers and molecules in order to modulate their interaction with the intestinal mucus.

\section{Enhancing mucoadhesion}

Different strategies to increase the mucoadhesion of nanocarriers have been followed, being the more frequently used those that rely on bioadhesive materials that interact with the mucus through different mechanisms, e.g. electrostatic interactions, hydrogen bounds, Van der Waals forces or covalent bounds. For that purpose, lipidbased nanocarriers have been modified with mucoadhesive materials. Chitosan, a polysaccharide derived from chitin, has been widely used for the formulation of nanocarriers due to its biocompatibility, biodegradability and bioadhesion. Chitosan-modified lipid-based nanocarriers have positive charge that facilitates the interaction with the intestinal mucus by electrostatic interactions, although Van der Waals forces, hydrogen and hydrophobic bonds can also be involved [192]. Additionally, chitosan can reversibly open tight junctions between enterocytes, what may enhance the paracellular transport and oral bioavailability [108, 193].

The group headed by M.J Alonso has greatly contributed to the development of chitosan-based nanocarriers [194-197]. Results from these studies led the authors to conclude that chitosan nanocapsules loaded with salmon calcitonin did not cross the Caco-2 cell monolayer, but remained at their apical side, showing preferable association to mucus secreting cells. Following oral administration to rats, they observed a prolonged and enhanced oral absorption of the peptide as well as higher hypocalcemic effect. Authors attributed the adhesive properties of chitosan to play a relevant role in the improved oral bioavailability of salmon calcitonin [194]. Shalaby et al. also used chitosan-coated nanocarriers to enhance the oral delivery of peptides. Concretely, they prepared chitosan-coated liposomes loaded with insulin. They observed that coated liposome adsorption to mucin was threefold higher compared with uncoated liposomes. This result was confirmed by an ex vivo intestinal mucoadhesion test, which showed that chitosan coated liposomes tended to accumulate in the mucus, opposite to uncoated liposomes.
Following intragastric administration, coated liposomes showed a prolonged hypoglycaemic effect compared with uncoated liposomes, what was probably due to their enhanced residence time in the intestinal mucosa achieved by chitosan [48]. In another study, Han et al. pointed out the ability of chitosan to eightfold increase liposome adsorption to mucin. They proposed these mucoadhesive liposomes as oral carriers of alendronate, reporting more than doubled increase in its oral bioavailability in rats, compared with free alendronate [21].

Chitosan is highly versatile in terms of chemical modification with different moieties like thiol groups. The thiolisation of chitosan promotes its interaction by disulphide bonds with the cysteine-rich residues of glycoproteins in the mucus [192]. This enhanced mucoadhesion may increase the intestinal residence time of nanocarriers and therefore boost their opportunity to interact with the intestinal epithelium, what may led to enhanced oral bioavailability of their cargo molecules [85, 108, 164]. This was observed by Vecchione et al. for iminothiol-modified chitosan nanocarriers, showing that the iminothiol degree of modification determines the oral performance of curcumin. Concretely, they reported a 33.2-fold increase in the oral bioavailability of the molecule in rats when high degree of iminothiol was used, compared with unformulated curcumin. Additionally, the co-encapsulation of piperine, inhibitor of the intestinal metabolism of curcumin, led to an increase in the oral bioavailability of curcumin by more than 64 times [85]. In a separated study, Gradauer et al. coupled thiolated chitosan to liposomes and reported an almost twofold increase in their intestinal mucoadhesion, compared with uncoated liposomes [198].

Chitosan has also been used in combination with other adhesive materials, as proposed by $\mathrm{Li}$ et al., who developed a multiple w/o/w nanoemulsion that included the adhesive polymer alginate and was further coated with chitosan. The oral administration of insulin-loaded nanoemulsions allowed a strong decrease of glucose levels, i.e. $60 \%$ from basal levels, in Goto-Kakizaki diabetic rats and a significant enhancement of the peptide bioavailability (8.19\%) compared with free insulin [199].

Despite the vast experience accumulated with chitosancoated nanocarriers, further polymers with the potential to adhere to mucus have been exploited, including hyaluronic acid, caprolactone, hydroxypropyl methylcellulose, alginate or pectin, among others [22, 83, 200-202]. Hyaluronic acid has negative charge and the potential to interact with the mucus via hydrogen bonds. Aguilera-Garrido et al. have recently developed lipid nanocapsules coated with hyaluronic acid that strongly interact with mucin. This interaction was higher when high molecular weight hyaluronic acid was used [22]. Cattani et al. developed 
adhesive caprolactone nanocapsules that behaved as an intestinal reservoir of a pro-drug of indomethacin, restraining its prompt metabolism in the intestinal lumen [200]. Pectin-coated liposomes loaded with calcitonin demonstrated strong mucoadhesion properties and were able to enhance the intestinal absorption of the peptide and to prolong its pharmacological effect, compared with uncoated liposomes, after oral administration to rats [202].

There are more factors that may affect to the mucoadhesion of nanocarriers that have been barely investigated and require further attention, such as the use of additional components to obtain the pharmaceutical dosage forms [23, 203]. It is also important to bear in mind that the biological modification of the adhesive shell of nanocarriers may change their properties and behaviour once administered. In this sense, further research is required regarding the potential metabolism of adhesive materials based on polysaccharides, e.g. chitosan or hyaluronic acid, by the intestinal microbiota, and the implications that this may have on the final mucoadhesiveness of the system.

\section{Enhancing mucodiffusion}

Mucodiffusive nanocarriers, also known as mucus penetrating nanocarriers, emerged as a strategy to improve the interaction with the epithelium and promote their absorption before being cleared by the mucus renewal. To facilitate the diffusion, nanocarriers must be able to minimize their interaction with the mucus and pass through the channels of the mucus matrix. The group headed by Hanes proposed coating nanocarriers with hydrophilic neutral polymers able to minimize the hydrophobic interactions with the mucus. Particularly, they proposed dense coatings with low molecular weight $(<10 \mathrm{kDa})$ PEG to yield mucodiffusive nanocarriers [191, 204, 205]. Since then, PEG has become the gold standard in the development of mucodiffusive nanocarriers [206]. Yuan et al. prepared PEGylated solid lipid nanoparticles and evaluated their permeation through Caco-2 and Caco-2/HT29 cell monolayers. The permeation of unmodified nanoparticles was higher in the Caco-2 monoculture but the incorporation of PEG to their surface improved their Caco-2/HT29 co-culture uptake rates, indicating the role played by mucus in the permeability of nanoparticles. The mucodiffusion capacity of PEGylated nanoparticles, confirmed by everted gut sac technique, led to enhanced oral bioavailability in rats of loaded doxorubicin, compared with the achieved by unmodified loaded nanoparticles [65].

A common strategy for the PEGylation of lipidbased nanocarriers is via non covalent bonds between the nanocarriers and PEG containing molecules, such as Pluronics ${ }^{\circledR}$ (Poloxamers) [206]. Pluronics ${ }^{\circledR}$ are amphiphilic polymers consisting of hydrophobic polypropyleneoxide (PPO) blocks and hydrophilic PEG chains. The PPO blocks allow the PEGylation of hydrophobic surfaces, and their number vary depending on the type of Pluronic $₫$, e.g. Pluronic ${ }^{\circledR}-\mathrm{F} 127$ (PF127, with 30 PPO units) or Pluronic ${ }^{\circledR}-\mathrm{F} 68$ (PF68, with 65 PPO units). On this basis, $\mathrm{Li}$ et al. reported that PF127-inlaid liposomes sevenfold enhanced the diffusion efficiency through intestinal rat mucus [207]. Chen et al. evaluated the mucodiffusion capacity of PF127-modified liposomes, compared with chitosan-modified liposomes. PF127-modified liposomes showed higher diffusion and penetration through intestinal rat mucus epithelium. As a result, in vivo studies showed enhanced oral absorption cyclosporine A when it was encapsulated in PF127-modified liposomes, compared with the chitosan-modified liposomes [56]. Mucodiffusive lipid carriers require dense Pluronic ${ }^{\circledR}$ coatings, as confirmed by Santalices et al. These authors studied the effect that the Pluronics ${ }^{\circledR}$ coating has on different nanocarriers (lecithin nanoemulsions and chitosan or polyarginine nanocapsules) with respect to their diffusion through intestinal mucus using particle tracking. PF127-coated nanoemulsions showed significant $(p<0.0001)$ enhanced mucodiffusion compared with uncoated nanoemulsions, what was indicative of dense PEGylation achieved by PF127. However, the presence of chitosan or polyarginine did not allow the formation of dense PF127 coatings of nanocapsules, and consequently, their diffusion was not enhanced [19].

As previously introduced, there is a current trend to balance mucoadhesion and mucodiffusion properties, in order to enhance the residence time of nanocarriers in the intestinal mucosa, but also to increase their opportunities to reach the epithelium. On this context, Liu et al. have recently developed liposomes functionalized with both thiolated chitosan and PF127. The multifunctional liposomes absorbed threefold more mucin compared with unmodified liposomes, due to chitosan functionalisation. Additionally, their intestinal penetration was enhanced compared with unmodified and chitosan-modified liposomes, due to PF127 functionalisation. This work showed the promising potential of nanocarriers with mucus adhesion and diffusion balance to increase the efficiency of oral drug delivery [208].

The diffusion of nanocarriers through the intestinal mucus is affected by their surface but also by their inner core. Santander et al. indicated that the hydrophobicity of the core determined the PF127 coating and subsequently the mucodiffusion of the system. They observed that the addition of $\alpha$-tocopherol, a highly hydrophobic molecule, to the oily core of nanoemulsions enhanced their mucodiffusion by 2 orders of magnitude, compared with less hydrophobic nanoemulsions, probably due to the achievement of denser PF127 coatings. This diffusion enhancement by PF127 
coating became more evident on polystyrene nanoparticles which higher hydrophobicity led to denser coatings, thus confirming the hypothesis of the authors [17].

The size of nanocarriers is another factor that may influence their diffusion through mucus. However, its impact on mucodiffusion may be difficult to assess since mucodiffusion depends on multiple factors, such as the surface chemistry or the intestinal contents [20]. In general terms, it is accepted that nanocarriers in the range of 200-500 nm have the potential to diffuse through mucus [ 23 , 209]. Shape may also impact the diffusion of nanocarriers. In particular, nonspherical nanocarriers, e.g. cylindric microemulsions or SMEDDS, could diffuse differently to spherically shaped nanocarriers, due to their distinct flow properties [117]. Nevertheless, the real impact of these parameters on mucodiffusion is still to be elucidated.

It is important to consider that the physicochemical properties of the nanocarriers, such as size, hydrophilicity or surface charge and composition, can be modified by the environment where they are immersed. In the oral route, a protein corona (PC) may be formed around the nanocarriers when they reach the complex gastrointestinal fluids [210]. Indeed, the gastrointestinal environment includes a wide variety of surface-active molecules that may coat ingested nanocarriers, such as enzymes, peptides, bile salts, lipids, food proteins and their digestion products [210]. The formation of the PC may transform nanocarriers and influence their oral performance, including their interaction with the mucus layer, as recently studied by Plaza et al. These authors pre-incubated mucoadhesive nanoemulsions with simulated gastrointestinal fluids including intestinal enzymes. The enzymatic coating, confirmed by transmission electronic microscopy, led to the reduction of the size of the nanoemulsion. Interestingly, this PC was able to significantly enhance the diffusion of the nanoemulsions. Indeed, the fraction of immobile particles decreased a $56 \%$, whereas freely diffusive particles experienced $20 \%$ increase. Authors observed a similar trend with indigestible polystyrene nanoparticles, confirming that the mucodiffusion enhancement was mainly due to the superficial PC, rather than to a possible size reduction by the enzyme action [18]. Similarly, Wang et al. prepared PC-coated liposomes by the adsorption of bovine serum albumin to liposomes. Coated liposomes showed effective mucus penetration, which was dramatically increased compared with uncoated liposomes. Concretely, particle tracking experiments revealed that the mean square displacement of coated liposomes was 21-fold increased. When insulin was encapsulated within coated liposomes, its oral delivery efficiency was increased in diabetic rats. Uncoated liposomes showed a $3.7 \%$ relative bioavailability, compared with subcutaneous insulin, whereas PC-coated liposomes achieved an $11.9 \%$, thereby confirming the impact of PC in the ability of nanocarriers to overcome gastrointestinal barriers and improve oral drug delivery [49].

\section{Inhibition of P-glycoprotein}

P-glycoprotein (Pgp) is an ATP-dependent transporter found in the apical surface of epithelial cells in the kidneys, intestine, placenta or endothelial brain cells. It has a protective function, identifying and expelling toxins and xenobiotics. Therefore, Pgp may play a relevant role in drug pharmacokinetics. Indeed, intestinal Pgp is responsible of the efflux from the enterocytes to the intestinal lumen of many drugs, including docetaxel, paclitaxel or saquinavir. This efflux decreases the chance that drugs might have to reach systemic circulation, and thereby, it has a negative impact on their oral bioavailability [211]. To solve this limitation, different strategies have been used to overcome or reduce the drug efflux, such as the coadministration of Pgp substrates with Pgp inhibitors. This approach has been also applied in nanotechnology, since a vast variety of nanocarriers, including lipid-based, incorporate at least one Pgp inhibitor in their composition.

Verapamil and cyclosporine have been traditionally used as Pgp inhibitors for improving the oral bioavailability of Pgp substrates. In particular, cyclosporine A (CsA) inhibits Pgp through a double mechanism: interfering with the substrate-binding sites and promoting ATP hydrolysis [211]. In this respect, Cui et al. have recently proposed the co-encapsulation of docetaxel and CsA into SNEDDS (DTX-CsA SNEDDS) for improving the oral bioavailability and efficacy of the anti-tumour drug. Interestingly, they observed a sequential drug release, being CsA released from the system prior to docetaxel probably by stronger interaction of docetaxel with the oily core of the SNEDDS. Both the intestinal permeability, assessed by in situ singlepass intestinal perfusion, and the plasma concentration of docetaxel were significantly higher $(p<0.05)$ in rats compared with those of docetaxel SNEDDS or solution (Fig. 5 a and b, respectively). Besides, oral DTX-CsA SNEDDS exhibited marked anti-tumour efficacy in 4T1 tumour-bearing mice, comparable with intravenous injection of docetaxel, as depicted in Fig. 5c, d [152].

The use of the classic Pgp inhibitors (verapamil and CsA) may be limited by their toxicity profile; therefore, alternative Pgp inhibitors have been proposed. Interestingly, there are indications that several surfactants commonly used in the formulation of nanocarriers can act as low-toxic Pgp inhibitors [211]. These excipients, like Cremophor, Tween 80 and PEG, inhibit the Pgp by altering the integrity of membrane lipids and the fluidity of the membrane. On this context, Yin et al. developed docetaxel-loaded 
a

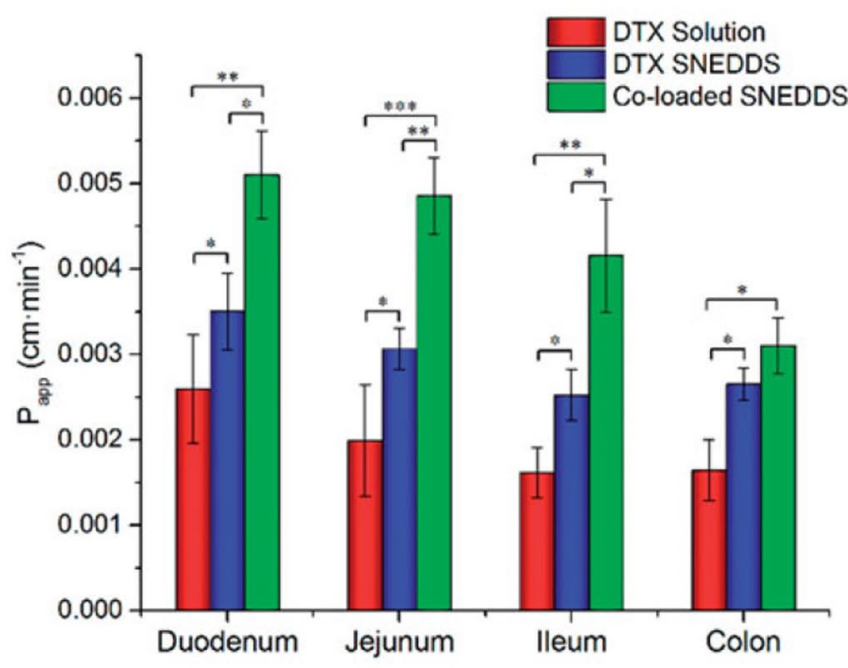

c

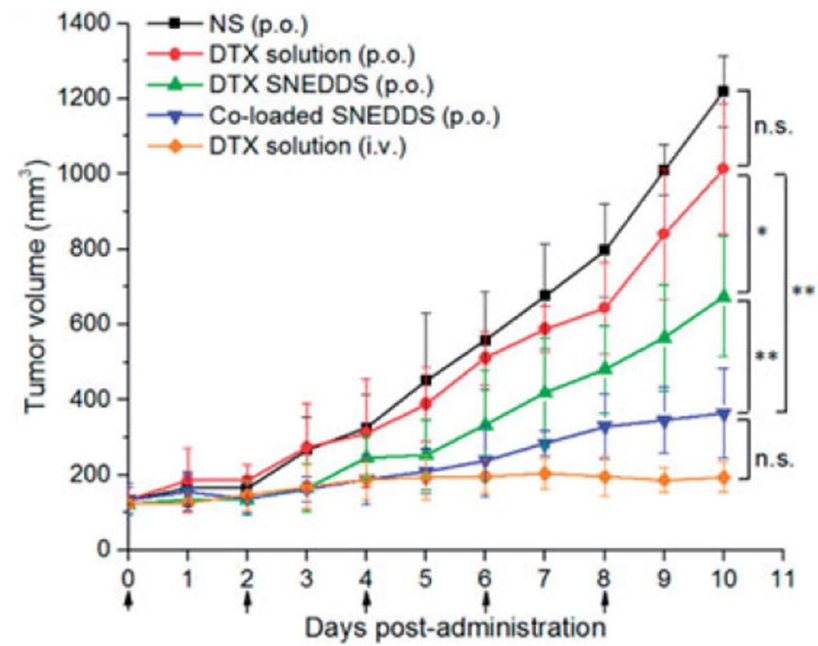

Fig. 5 a Permeability of co-loaded SNEDDS across different intestinal sections. b Docetaxel plasma concentrations after the different treatments: oral administration of DTx solution, DTX SNEDDS or co-loaded SNEDDS, or intravenous administration of DTX solution to rats. c Tumour volume in 4T1 tumour-bearing mice following each

microemulsions that were mainly stabilised by Cremophor and studied their impact on the Pgp-mediated efflux of docetaxel by Caco-2 cells, which also express Pgp in their apical membrane. Authors reported a significant increase of docetaxel permeability and a decrease in the drug efflux from Caco- 2 cells when administered in microemulsions compared with Taxotere ${ }^{\circledR}$, showing more than fivefold increase in docetaxel oral bioavailability after the administration of the formulation to rats [123]. Similarly, the oral administration of andrographolide, a Pgp substrate, encapsulated in Cremophor-stabilised nanoemulsions strongly increased the oral bioavailability of the drug in rats. This improved the in vivo anti-inflammatory efficacy of the formulation to rats with indomethacin-induced intestinal lesions, significantly b

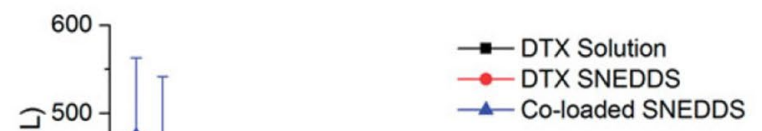

d

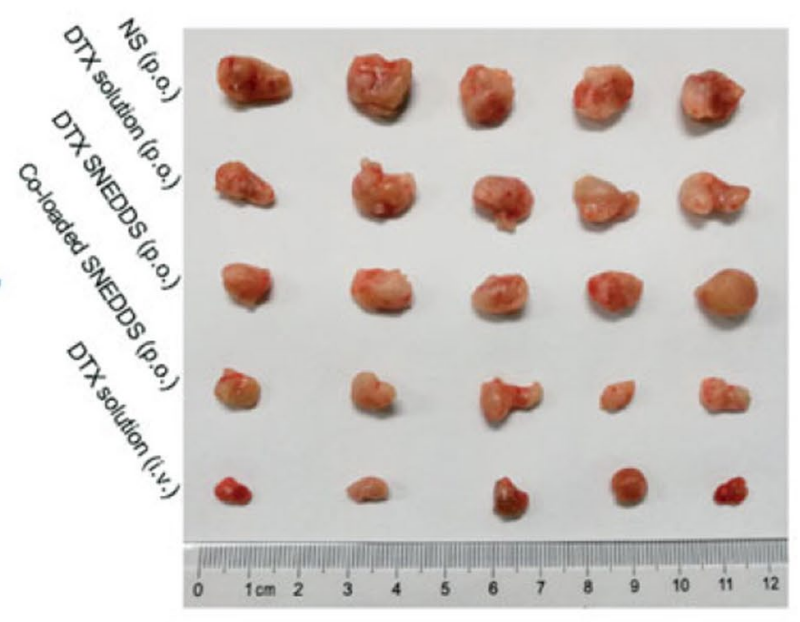

treatment. d Images of tumours. $* p<0.05, * * p<0.01$, $* * * p<0.001$ ). Data are presented as mean \pm standard deviation. DTX: docetaxel; coloaded SNEDDS: SNEDDs loaded with docetaxel and cyclosporine A; p.o.: oral; i.v.:intravenous. Adapted with permission of [152]

reducing the ulcer index and the histological intestinal damage [212]. The ability of Tween 80 (polysorbate) to inhibit Pgp has also shown a promising role in the bioavailability enhancement of Pgp substrates [149, 156, 213]. In this line, Goo et al. observed 4.1-fold permeation enhancement of valsartan, a Pgp substrate, through rat jejunum by its encapsulation in SNEDDS stabilized by Tween 80 , as well as a $470 \%$ increase in its oral bioavailability in rats. Authors pointed the Pgp inhibition by Tween 80 to play a relevant role in these improvements [149]. Similarly, Zhou et al. have recently exploited the potential of polysorbate- 80 to inhibit drug efflux. These authors reported higher intestinal permeability of tilmicosin-loaded solid lipid nanoparticles when polysorbate-80 was added as Pgp inhibitor [213]. 
Additional molecules routinely used in the formulation of lipid-based nanocarriers that have shown promise as intestinal Pgp inhibitors include D- $\alpha$-tocopheryl polyethylene glycol succinate (TPGS) and deoxycholic acid $[81,89,135,156]$. Vyas et al. developed o/w nanoemulsions containing saquinavir that incorporated deoxycholic acid in their aqueous phase. They observed a significant $(p<0.05)$ increase in the oral bioavailability of saquinavir when it was delivered to mice in nanoemulsions, compared with aqueous suspension, suggesting that the inhibition of saquinavir efflux by Pgp could have contributed to improve its oral performance [81].

Natural compounds like myricetin, curcumin or quercetin have also demonstrated potential for the inhibition of Pgp [211]. In this line, Jain et al. reported an eightfold increase in the oral bioavailability of tamoxifen, a Pgp substrate, when quercetin was co-encapsulated into SNEDDS, achieving higher anti-tumour effect in tumour-bearing mice. Besides, quercetin contributed to lower the hepatotoxicity of tamoxifen [153]. A similar strategy was proposed by Singh et al., who exploited the ability of curcumin to inhibit Pgp efflux to enhance the oral bioavailability of paclitaxel. They encapsulated both molecules within SNEDDS containing dietary lipids and observed that the intestinal permeability and the oral bioavailability of paclitaxel was enhanced compared with SNEDDS without curcumin or to paclitaxel solution [155]. In this line, Nasirizadeh et al. have recently reported higher cytotoxicity in Caco-2 cells of solid lipid nanoparticles loaded with the antitumour drug sn38 when piperine and quercetin were added as Pgp inhibitors. Studies performed in tumour-bearing mice showed an enhanced survival time when animals received the oral combination treatment ( $\operatorname{sn} 38$ nanoparticles, piperine and quercetin), compared with free sn38 and to sn38 nanoparticles without these Pgp inhibitors [66].

\section{Targeting the intestinal mucosa}

Intestinal cells present a wide variety of transporters and receptors in their membranes that interact with specific ligands, e.g. vitamins or hormones. Taking advantage of such receptors and transporters, active targeting implies the surface functionalisation of nanocarriers with ligands able to enhance their interaction with particular cell populations [214]. On this basis, different targeted lipidbased nanocarriers have been proposed in the last years as a potential strategy to enhance their intestinal internalisation and therefore their chances to reach the systemic circulation.

The surface modification of nanocarriers with vitamins, such as folic acid $\left(B_{9}\right)$, biotin $\left(B_{7}\right)$ or thiamine $\left(B_{1}\right)$, has been used to target enterocytes, with the aim to mimic the intestinal absorption pathways of the vitamins, and hence facilitate the absorption of nanocarriers. Folic acid is internalised by enterocytes following receptor-mediated endocytosis. It has been deeply exploited as target ligand of oral nanocarriers, due to its high biocompatibility and affinity to folic acid receptors, which are expressed at the apical membrane of enterocytes. Ling et al. demonstrated that cefotaxime-loaded liposomes functionalised with folic acid were able to significantly increase the AUC of cefotaxime after their oral administration to rats, compared with folic acid-free loaded liposomes [215]. In this line, Agrawal et al. developed insulin-loaded liposomes and decorated their surface with folic acid by multilayer electrostatic deposition. In vivo studies revealed an achievement of $20 \%$ insulin relative oral bioavailability, compared with subcutaneous insulin [51]. Similarly, in the intestine, biotin can be transported through sodiumdependent multivitamin transporter and by the biotin receptor. To exploit this target, Zhang et al. developed biotinmodified liposomes loaded with insulin. These authors reported an enhanced oral bioavailability of insulin after their oral administration to diabetic mice, which doubled that of non-modified liposomes [53]. The potential of vitamins-decorated liposomes as targeted drug carriers has been recently confirmed by He et al., who have shown that insulin-loaded liposomes decorated with thiamine or niacin are able to achieve a sustained hypoglycemic effect after their oral administration in rats, superior to that achieved by conventional liposomes [50].

In addition to vitamins, some peptides have also been described as ligands to target the intestinal mucosa, such as lectins (agglutinins), which show strong affinity to carbohydrates attached to lipids or proteins. The rationale behind the selection of lectins as targeting ligands relies on the glycosylated lipids and proteins that are highly present in cellular membranes [64, 216]. Concretely, wheat germ agglutinin has been used to target the intestinal mucosa, since it binds to $\mathrm{N}$-acetyl-D-glucosamine and sialic acid residues, found at the intestinal cell surface [214]. Considering this ligand-target interaction, Pooja et al. prepared paclitaxelloaded solid lipid nanoparticles conjugated with wheat germ agglutinin. This nanocarrier turned to enhance the oral bioavailability of paclitaxel in rats. Besides, nonspecific toxicity of paclitaxel was decreased, since it accumulated preferentially in the lungs, opposite to free paclitaxel, which tended to accumulate in the liver [64].

Not only enterocytes, but further intestinal cells can be targeted, such as goblet cells or M cells [214]. The peptide CSKSSDYQC (CSK) has been described to target goblet cells [217]. Tian et al. developed atorvastatin calcium-loaded solid lipid nanoparticles whose surface was modified with CSK. Compared with unmodified nanoparticles, CSK-modified nanoparticles showed a more efficient uptake across Caco2/HT29 cell monolayer. It was also demonstrated, using an in situ perfusion method in 
rats, that CSK-modified nanoparticles showed significant higher intestinal permeability, compared with free atorvastatin calcium [218]. M cells may act as an entrance for microorganisms, and they are involved in the start of the antigen-specific immune responses. Therefore, their targeting through appropriate lipid-based nanocarriers has arisen as an appealing alternative to conventional vaccination by the parental route. In this line, Gupta et al. encapsulated hepatitis B surface antigen into liposomes coupled with lectin. Authors reported higher immune response and antibodies levels in mucosal secretions in mice after oral administration of lectin-functionalised loaded liposomes, compared with unmodified loaded liposomes. Authors attributed this effect to the $\mathrm{M}$ cells targeting and longer persistence of antigen in Peyer's patches achieved by lectin-functionalised liposomes [219].

Opposite to the surface engineering of nanocarriers for cell targeting, there is a current approach based on exploiting the components used for the preparation of nanocarriers as targeting ligands themselves. In this line, the group headed by V. Préat proposed an innovative approach for targeting enteroendocrine L cells, which secrete hormones such as glucagon-like peptide-1 (GLP-1). This peptide stimulates insulin secretion, showing potential for type 2 diabetes treatment. L cells present at their apical membrane receptors that could be activated by dietary nutrients, such as lipids or proteins. In this respect, these authors introduced the concept that lipid-based nanocarriers may act as endogenous ligands to stimulate GLP-1 secretion from L cells, without needing a surface-ligand-modified system [220]. A study from the same group evaluated the size effect of lipid nanocapsules on GLP-1 secretion from murine L cells, reporting that $200 \mathrm{~nm}$ but not 25-150 nm nanocapsules were able to increase GLP-1 secretion by GLUTag cells, an enteroendocrine cell model. This work prompts the authors to conclude that the stimulation of GLP-1 secretion by L cells achieved by nanocapsules was size dependent [221]. However, the short half-life of GLP-1 suggested the use of other GPL-1 analogues, such as exenatide, as an alternative molecule. On this context, $\mathrm{Xu}$ et al. have recently developed dual-action reverse micelle nanocapsules loaded with exenatide for the treatment of type 2 diabetes. Unloaded nanocapsules, sized $220 \mathrm{~nm}$, were able to stimulate GLP-1 secretion both in human and murine $\mathrm{L}$ cells and in normoglycaemic mice, as shown in Fig. 6a, b. Besides, exenatide encapsulation led to a fourfold enhancement on its oral bioavailability, compared with an exenatide solution (Fig. 6c). Interestingly, the combination of increased GLP-1 secretion and increased exenatide bioavailability was able to achieve normal glycemia of obese/diabetic mice after 5 weeks of daily oral administration (Fig. 6d), as well as to reduce levels of inflammatory markers associated with type 2 diabetes. [131].
The targeting strategy based on unmodified surface nanocarriers has also been recently followed by Plaza et al. These authors have developed nanoemulsions stabilized by ascorbyl-dipalmitate, an amphiphilic surfactant that derives from ascorbic acid, with the aim to target the ascorbic acid transporter (SVCT-1). This transporter is expressed at the apical brush of both enterocytes and differentiated Caco- 2 cells. Authors reported that the nanoemulsions were taken up and retained within Caco- 2 cells. The cellular internalisation decreased after pre-incubating the cells with ascorbic acid at the SVCT-1 saturation range, indicating that the transporter could be involved in the uptake of the nanoemulsion. The developed nanoemulsion thereby showed promise as a targeted carrier able to deliver drugs to the intestinal mucosa with no need of surface modification. Authors confirmed this potential by the preparation of curcumin-loaded nanoemulsions, which were able to deliver curcumin within Caco- 2 cells, where it fourfold reduced ROS levels, compared with a curcumin suspension [30].

\section{Enhancement of lymphatic transport}

The lymphatic system is a complex network of conducts that reabsorbs the extracellular fluid to maintain the water balance of the body and plays a major role in the oral absorption of macromolecules such as dietary lipids or insoluble vitamins. It drains directly into the systemic circulation, avoiding the hepatic first pass [222, 223]. In this transport system, the carriers are chylomicrons, which are lipoproteins showing the lowest density, mainly composed of triglycerides.

Lipid-based nanocarriers, particularly those including unsaturated long chain fatty acids, have the potential to enhance the synthesis of chylomicrons and the lymphatic transport of their hydrophobic cargo molecules [222, 223]. Besides, excipients commonly used in the formulation of lipid-based nanocarriers, e.g. phospholipids, Tween 80 or TPGS, may provide an additional enhancement of the lymphatic transport, as assessed in a recent study by Liao et al. These authors developed SMEDDS stabilized by Tween 80 and loaded with the flavonoid baicalain. The in vivo evaluation in rats indicated that baicaleinloaded SMEDDS showed a relative oral bioavailability of $342.5 \%$, compared with free drug. These levels were further increased to 448.7 when a phospholipid complex of baicalein was used. In order to investigate the role played by the lymphatic system in this enhancement, authors also used a chylomicrons flow blocking rat model, i.e. pre-treated with cycloheximide. Interestingly, this study revealed that $<20 \%$ of orally absorbed free 
a
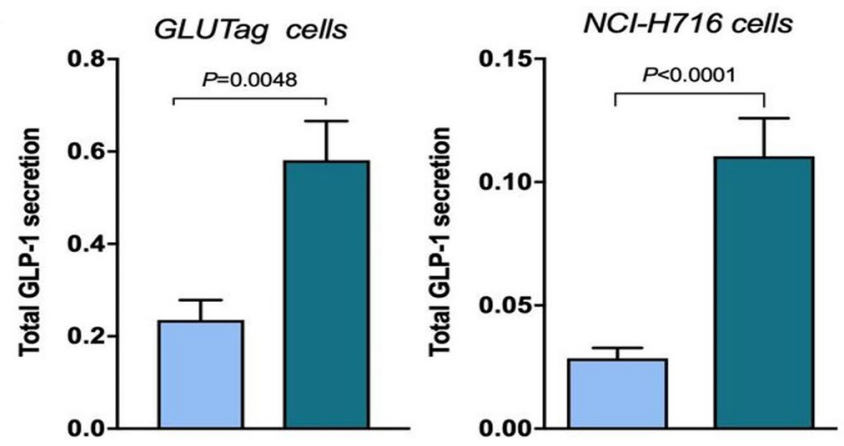

b

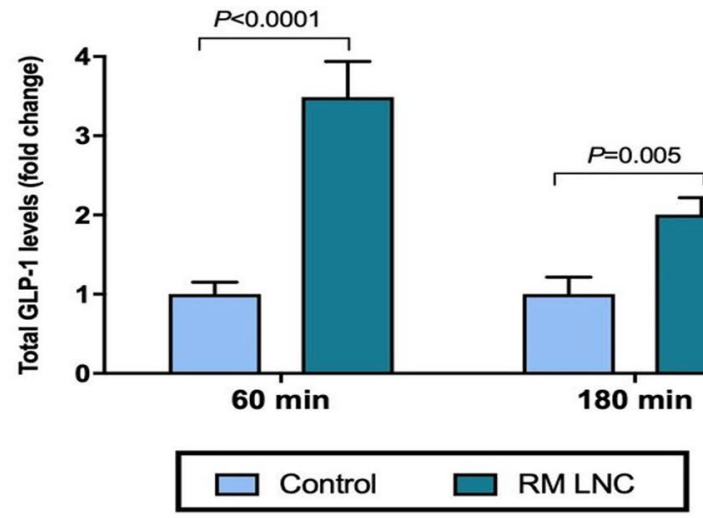

d

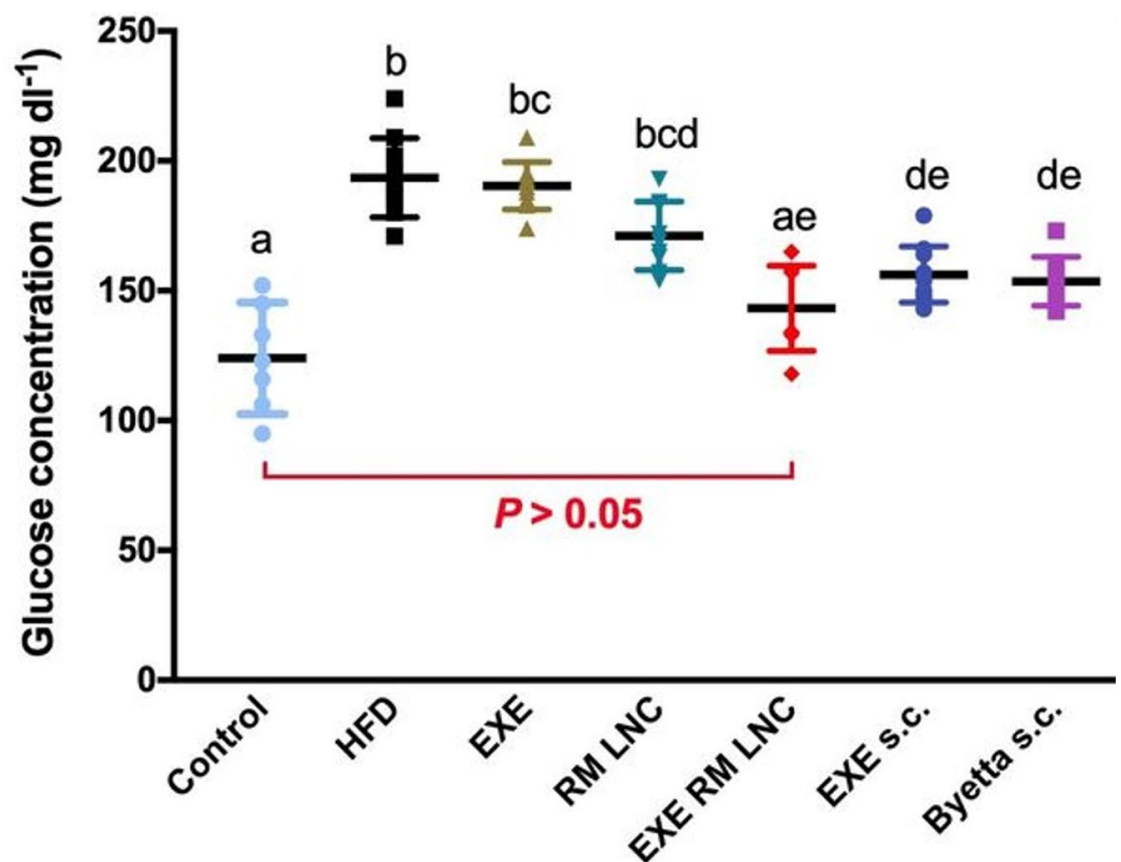

c

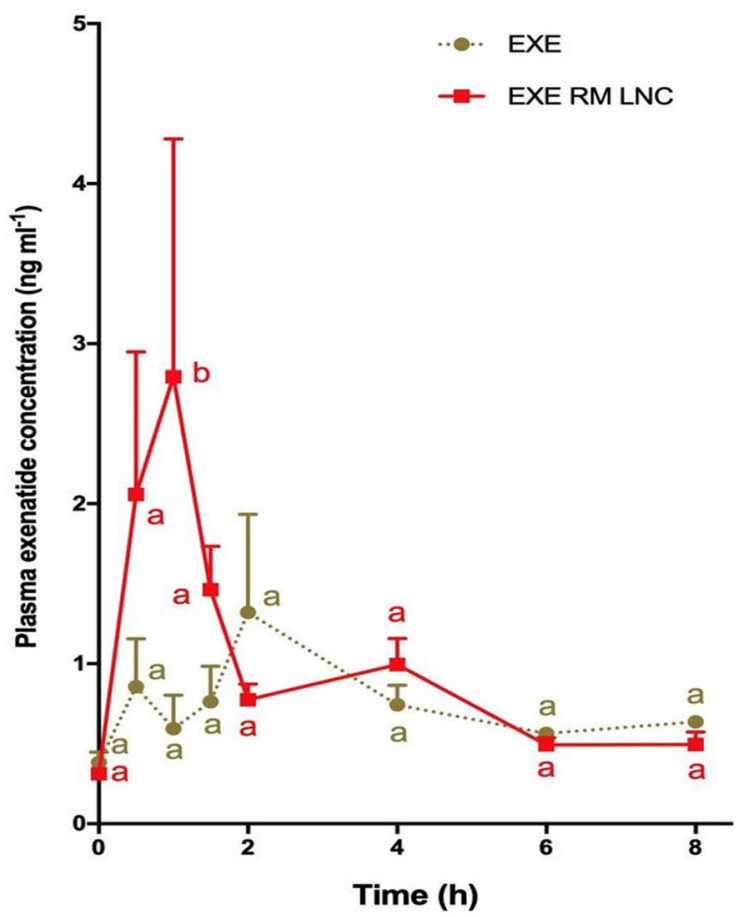

Time (h)
Fig. 6 a GLP-1 secretion in GLUTag and NCI-H716 cells (murine and human $\mathrm{L}$ cells, respectively) after a $2 \mathrm{~h}$ incubation period with RM-LNC $(n=6-10)$. b GLP-1 levels detected 60 or 180 min after the oral administration of RM LNC to normoglycaemic mice $(n=7-8)$. c Plasmatic exenatide concentration after the oral administration of EXE RM LNC to normoglycaemic mice, compared with a solution of EXE $(n=4)$. d Plasma glucose levels after 5 weeks of oral treatment, showing the effect of EXE RM LNC on glucose homeostasis in type 2 diabetic mice (13 weeks of HFD feeding) $(n=10)$. Data are presented as the mean \pm SEM. EXE: exenatide; RM LNC: reverse micelle lipid nanocapsules; EXE RM LNC: exenatide reverse micelle lipid nanocapsules; HFD: high-fat diet; EXE s.c.: subcutaneous exenatide; Byetta s.c.: subcutaneous administration of a marketed form of exenatide. Adapted with permission of [131] 
baicalein entered the systemic circulation through the lymphatic system, whereas this transport, respectively, increased to $>56$ and $>70.2 \%$ after the administration of SMEDDS loaded with the flavonoid or its phospholipid complex. Taken together, these results revealed the ability that phospholipids and SMEDDS stabilized by Tween 80 have to enhance the lymphatic uptake of poor-water soluble molecules [173]. Sangsen et al. reported an increase of the oral bioavailability of oxyresveratrol by its encapsulation in SMEDDS, which was significantly higher than the improvement observed when Labrasol was used as surfactant. Authors hypothesized that the lymphotropic properties of Tween 80 and the resulting bypass of the hepatic first pass could contribute to the enhanced oral bioavailability of SNEDDS loaded with oxyresveratrol [167]. Similar conclusions were reported by Patel et al. in a recent study. These authors prepared solid lipid nanoparticles that included TPGS in their formulation to increase the oral bioavailability of asenapine maleate, a drug which undergoes extensive first pass metabolism. They reported a 50-fold improvement in the oral bioavailability of asenapine maleate in rats, compared with free drug. The plasmatic concentration of the drug significantly decreased when animals were pre-treated with cycloheximide, unveiling the contribution of lymphatic transport in the enhanced drug bioavailability [68].

The lymphatic system has a relevant role in the metastasis of solid tumours, and it is also a transport pathway for lymphocytes, being associated with the development and spread of immunodeficiency virus (HIV) or hepatitis B and $\mathrm{C}$ virus, among others. For this reason, achieving an enhanced lymphatic transport may be especially desirable for anti-tumour, antiretroviral and immunomodulatory drugs [224-227]. Garg et al. developed solid SNEDDS stabilized by Tween 80 and loaded with lopinavir. After their oral administration to rats, they observed an enhancement of the oral bioavailability of lopinavir, showing AUC values 3.9fold higher compared with the free drug. Cycloheximidepre-treated rats experienced a significant decrease in the oral absorption of lopinavir, indicating the ability of SMEDDS to enhance the lymphatic uptake of the antiretroviral drug. Since the lymphatic system is the main reservoir of HIV, the proposed SMEDDS showed great promise for development of future of antiretroviral therapies [225]. In this line, Makwanaa et al. developed solid lipid nanoparticles to improve the oral delivery of efavirenz. Following oral administration of efavirenz-loaded nanoparticles in rats, authors reported reduced hepatic concentrations of the drug, a fact that contributed to increase its bioavailability, since efavirenz undergoes extensive first pass effect. Opposite, drug hepatic concentrations were higher in the case of rats pre-treated with cycloheximide [71].
Apart from the chylomicron uptake, drugs can reach lymphatic circulation via GALT from $M$ cells, although the former is thought to be the major mechanism [228]. Ge et al. developed nanoemulsions loaded with a protein complex including a tumour antigen. After their oral administration, loaded nanoemulsions elicited an immune response in tumour-bearing mice that was similar to the immune response observed when nanoemulsions were administered by the parenteral route. Authors hypothesized that this effect could be explained by the intestinal lymphatic uptake of the nanoemulsion at $M$ cells [224]. In this sense, achieving targeting to $\mathrm{M}$ cells may also be interesting to enhance the lymphatic transport of lipid-based nanocarriers.

\section{Conclusions}

During the last two decades, there has been an intense and increased research focused on lipid-based nanocarriers as oral drug delivery systems. This has been boosted by their potential to improve oral drug stability, solubility and intestinal permeability together with a low toxicity profile. However, despite the advances achieved and the increasing number of filed patents in this field, only a limited number of oral lipid-based nanocarriers have reached clinical trials and few of them have been approved for clinical use. They face many obstacles in their way to clinical translation, but the poor in vitro-in vivo correlation plays a major role. The gastrointestinal tract entails a harsh and complex environment difficult to replicate by the conventional models available. Additionally, the gastrointestinal tract includes physicochemical barriers to oral drug delivery, such as extreme and variable $\mathrm{pH}$, enzymes or mucus, among others. Interestingly, these extreme conditions that are challenges for lipid-based nanocarriers may be turned into opportunities if they are designed considering them predicting the biological behaviour of the systems. For this purpose, a rational selection of the materials used to formulate the nanocarriers according to the strategy aimed is mandatory. The knowledge that we currently have and the advances towards more refined techniques have promoted the great progress observed in lipid-based nanocarriers for oral delivery.

\section{Future remarks}

In last the years, it has been well understood that oral nanocarriers require a design and development based on the real gastrointestinal conditions that they will encounter at the gastrointestinal tract. However, there are still some characteristics of the gut that have been overlooked when 
developing nanocarriers, like the gut microbiota and its effects on the gut physiology, pathology and metabolism of nanocarriers, as well as the role of enzymes on their performance. In-depth knowledge about the plausible gut-nanocarrier interactions may contribute to a better understanding of the role played by the surface of lipidnanocarriers and, consequently, to the development of more active targeted lipid-based nanocarriers in the future. On this basis, the progress of in vitro models towards more sophisticated and bioinspired prototypes is a must for the success of oral lipid-based nanocarriers. In this regard, 3D cell culture based on microfluidics devices, e.g. gut on a chip, is expected to grow in the near future for the evaluation of nanocarriers. These systems are able to recapitulate the complex gastrointestinal environment, including gut microbiota, mucus and peristaltic movements, so they are predicted to play a relevant role in the development of novel bioinspired lipid-based nanocarriers in the near and mid-term future. The implementation of these techniques will have a clear impact on the clinical translation of these technologies, helping to gradually narrow the current gap between academic research and industrial development of lipidbased nanocarriers in the next years.

Funding Authors received financial support provided by the University of Castilla-La Mancha (UCLM) (01110AB026 and 01110AB039). M. Plaza received financial support given by the UCLM 10 (OE154 and 2018/152504).

\section{Compliance with ethical standards}

Conflict interests The authors declare that they have no competing interests.

Ethics approval and consent to participate Graphical abstract and Figs. 1 and 4 have been created through BioRender.com. Figures reproduced from another works count with the proper permissions. Ethical approval and informed consent are not applicable.

\section{References}

1. Obeid MA, Tate RJ, Mullen AB, Ferro VA. Lipid-based nanoparticles for cancer treatment. Lipid Nanocarriers Drug Target. Elsevier Inc. 2018;313-59.

2. Niu Z, Conejos-Sánchez I, Griffin BT, O’Driscoll CM, Alonso MJ. Lipid-based nanocarriers for oral peptide delivery. Adv Drug Deliv Rev. 2016;106:337-54.

3. Feeney OM, Crum MF, McEvoy CL, Trevaskis NL, Williams HD, Pouton CW, et al. 50 years of oral lipid-based formulations: Provenance, progress and future perspectives. Adv Drug Deliv Rev. Elsevier B.V. 2016;101:167-94.

4. Pitcher SN, Clinton D. Oral anaerobic glutathione supplement in liposome suspension. United States Patent US 10272130B1. $2018 ; 1$.
5. UNIVERSITESI TCE. Self-micro/ nanoemulsifying drug carrying system for oral use of rosuvastatin. Patent WO 2015/142307Al. 2015.

6. Alonso MJ, Santander-Ortega M, Zhigao N. Nanoparticles with protected interiors, and methods of use thereof. Patent US10471020B2. 2016.

7. Kidron M. Methods and compositions for oral administration of proteins. Patent WO2007029238A2. 2007;2007.

8. https://www.oramed.com/ (Consulted 231220).

9. Homayun B, Lin X, Choi HJ. Challenges and recent progress in oral drug delivery systems for biopharmaceuticals. Pharmaceutics. 2019;11.

10. Kiptoo P, Calcagno AM, Siahaan TJ. Physiological, biochemical, and chemical barriers to oral drug delivery. In: Binghe, Wang; Longqin Hu and TJS, editor. Drug Deliv Princ Appl. Second. John Wiley \& Sons, Inc. 2016. p. 19-34.

11. Zhang R, McClements DJ. Characterization of gastrointestinal fate of nanoemulsions. Nanoemulsions Formul Appl Charact. Elsevier Inc. 2018;577-612.

12. Lozano MV, Santander-Ortega MJ, Alonso MJ. In vitro relevant information for the assessment of nanoparticles for oral drug administration. Nanotechnol oral drug Deliv. 2020. p. 419-58.

13. Jantratid E, Janssen N, Reppas C, Dressman JB. Dissolution media simulating conditions in the proximal human gastrointestinal tract: an update. Pharm Res. 2008;25:1663-76.

14. Jantratid E, Janssen N, Chokshi H, Tang K, Dressman JB. Designing biorelevant dissolution tests for lipid formulations: case example - LIpid suspension of RZ-50. Eur J Pharm Biopharm. 2008;69:776-85.

15. Ensign LM, Cone R, Hanes J. Oral drug delivery with polymeric nanoparticles: the gastrointestinal mucus barriers. Adv Drug Deliv Rev. 2012;64:557-70.

16. Lock JY, Carlson TL, Carrier RL. Mucus models to evaluate the diffusion of drugs and particles. Adv Drug Deliv Rev. 2018;124:34-49.

17. Santander-Ortega MJ, Plaza-Oliver M, Rodríguez-Robledo V, Castro-Vázquez L, Villaseca-González N, González-Fuentes $\mathrm{J}$, et al. PEGylated nanoemulsions for oral delivery: role of the inner core on the final fate of the formulation. Langmuir. 2017;33:4269-79.

18. Plaza-Oliver M, Santander-Ortega MJ, Castro-Vázquez L, Rodríguez-Robledo V, González-Fuentes J, Marcos P, et al. The role of the intestinal-protein corona on the mucodiffusion behaviour of new nanoemulsions stabilised by ascorbyl derivatives. Colloids Surfaces B Biointerfaces. Elsevier; 2020;186:110740.

19. Santalices I, Torres D, Lozano MV, Arroyo-Jiménez MM, Alonso MJ, Santander-Ortega MJ. Influence of the surface properties of nanocapsules on their interaction with intestinal barriers. Eur J Pharm Biopharm Elsevier. 2018;133:203-13.

20. Yildiz HM, McKelvey CA, Marsac PJ, Carrier RL. Size selectivity of intestinal mucus to diffusing particulates is dependent on surface chemistry and exposure to lipids. J Drug Target. 2015;23:768-74.

21. Han HK, Shin HJ, Ha DH. Improved oral bioavailability of alendronate via the mucoadhesive liposomal delivery system. Eur J Pharm Sci. Elsevier B.V. 2012;46:500-7.

22. Aguilera-Garrido A, Molina-Bolívar JA, Gálvez-Ruiz MJ, Galisteo-González F. Mucoadhesive properties of liquid lipid nanocapsules enhanced by hyaluronic acid. J Mol Liq. 2019;296.

23. Das Neves J, Bahia MF, Amiji MM, Sarmento B. Mucoadhesive nanomedicines: characterization and modulation of mucoadhesion at the nanoscale. Expert Opin Drug Deliv. 2011;8:1085-104.

24. Thursby E, Juge N. Introduction to the human gut microbiota. Biochem J. 2017;474:1823-36. 
25. Wilkinson EM, Ilhan ZE, Herbst-Kralovetz MM. Microbiota-drug interactions: impact on metabolism and efficacy of therapeutics. Maturitas. 2018;112:53-63.

26. Karavolos M, Holban A. Nanosized drug delivery systems in gastrointestinal targeting: interactions with microbiota. Pharmaceuticals. 2016;9:1-15.

27. Chen H, Wang B, Gao D, Guan M, Zheng L, Ouyang H, et al. Broad-spectrum antibacterial activity of carbon nanotubes to human gut bacteria. Small. 2013;9:2735-46.

28. Peterson LW, Artis D. Intestinal epithelial cells: regulators of barrier function and immune homeostasis. Nat Rev Immunol Nature Publishing Group. 2014;14:141-53.

29. Beloqui A, des Rieux A, Préat V. Mechanisms of transport of polymeric and lipidic nanoparticles across the intestinal barrier. Adv Drug Deliv Rev. Elsevier B.V. 2016;106:242-55.

30. Plaza-Oliver M, Beloqui A, Santander-Ortega MJ, CastroVázquez L, Rodríguez-Robledo V, Arroyo-Jiménez MM, et al. Ascorbyl-dipalmitate-stabilised nanoemulsions as a potential localised treatment of inflammatory bowel diseases. Int J Pharm. Elsevier. 2020;586:119533

31. Artursson P, Palm K, Luthman K. Caco-2 monolayers in experimental and theoretical predictions of drug transport. Adv Drug Deliv Rev. 2001;64:280-9.

32. Walter E, Janich S, Roessler BJ, Hilfinger JM, Amidon GL. HT29MTX/Caco-2 cocultures as an in vitro model for the intestinal epithelium: in vitro-in vivo correlation with permeability data from rats and humans. J Pharm Sci. 1996;85:1070-6.

33. Gullberg E, Leonard M, Karlsson J, Hopkins AM, Brayden D, Baird AW, et al. Expression of specific markers and particle transport in a new human intestinal M-cell model. Biochem Biophys Res Commun. 2000;279:808-13.

34. des Rieux A, Fievez V, Théate I, Mast J, Préat V, Schneider YJ. An improved in vitro model of human intestinal follicleassociated epithelium to study nanoparticle transport by $\mathrm{M}$ cells. Eur J Pharm Sci. 2007;30:380-91.

35. Antunes F, Andrade F, Araújo F, Ferreira D, Sarmento B. Establishment of a triple co-culture in vitro cell models to study intestinal absorption of peptide drugs. Eur J Pharm Biopharm. 2013;83:427-35.

36. Sung JH, Yu J, Luo D, Shuler ML, March JC. Microscale 3-D hydrogel scaffold for biomimetic gastrointestinal (GI) tract model. Lab Chip. 2011;11:389-92.

37. Kim HJ, Huh D, Hamilton G, Ingber DE. Human gut-on-achip inhabited by microbial flora that experiences intestinal peristalsis-like motions and flow. Lab Chip. 2012;12:2165-74.

38. Jalili-Firoozinezhad S, Gazzaniga FS, Calamari EL, Camacho DM, Fadel CW, Bein A, et al. A complex human gut microbiome cultured in an anaerobic intestine-on-a-chip. Nat Biomed Eng. Springer US 2019;3:520-31.

39. Shrestha, Neha, Préat V. In vivo testing of orally delivered nanoparticles. Nanotechnol oral drug Deliv. 2020. p. 459-80.

40. Costa, Joana, Ahluwalia A. Advances and current challenges in intestinal in vitro model engineering: a digest. Front Bioeng Biotechnol. 2019;7:1-14.

41. Gamboa JM, Leong KW. In vitro and in vivo models for the study of oral delivery of nanoparticles. Adv Drug Deliv Rev. Elsevier B.V. 2013;65:800-10.

42. Lee MK. Liposomes for enhanced bioavailability of waterinsoluble drugs: In vivo evidence and recent approaches. Pharmaceutics. 2020;12.

43. Nguyen TX, Huang L, Gauthier M, Yang G, Wang Q. Recent advances in liposome surface modification for oral drug delivery. Nanomedicine. 2016;11:1169-85.

44. Bangham AD, Standish MM, Watkins JC. Diffusion of univalent ions across the lamellae of swollen phospholipids. J Mol Biol. Academic Press Inc. (London) Ltd. 1965;13:238-52.
45. Bhatt P, Lalani R, Vhora I, Patil S, Amrutiya J, Misra A, et al. Liposomes encapsulating native and cyclodextrin enclosed paclitaxel: Enhanced loading efficiency and its pharmacokinetic evaluation. Int J Pharm [Internet]. Elsevier 2018;536:95-107. Available from: https://doi.org/10.1016/j. ijpharm.2017.11.048

46. Deng W, Chen W, Clement S, Guller A, Zhao Z, Engel A, et al. Controlled gene and drug release from a liposomal delivery platform triggered by X-ray radiation. Nat Commun [Internet]. Springer US; 2018;9:1-11. Available from: https:// doi.org/10.1038/s41467-018-05118-3

47. Liang J, Wu W, Liu Q, Chen S. Long-circulating nanoliposomes (LCNs) sustained delivery of baicalein (BAI) with desired oral bioavailability in vivo. Drug Deliv. 2013;20:319-23.

48. Shalaby TI, El-Refaie WM. Bioadhesive chitosan-coated cationic nanoliposomes with improved insulin encapsulation and prolonged oral hypoglycemic effect in diabetic mice. J Pharm Sci American Pharmacists Association. 2018;107:2136-43.

49. Wang A, Yang T, Fan W, Yang Y, Zhu Q, Guo S, et al. Protein corona liposomes achieve efficient oral insulin delivery by overcoming mucus and epithelial barriers. Adv Healthc Mater. 2019;8:1-11.

50. He H, Lu Y, Qi J, Zhao W, Dong X, Wu W. Biomimetic thiamine- and niacin-decorated liposomes for enhanced oral delivery of insulin. Acta Pharm Sin B. 2018;8:97-105.

51. Agrawal AK, Harde H, Thanki K, Jain S. Improved stability and antidiabetic potential of insulin containing folic acid functionalized polymer stabilized multilayered liposomes following oral administration. Biomacromol. 2014;15:350-60.

52. Zhang N, Ping QN, Huang GH, Xu WF. Investigation of lectinmodified insulin liposomes as carriers for oral administration. Int J Pharm. 2005;294:247-59.

53. Zhang X, Qi J, Lu Y, He W, Li X, Wu W. Biotinylated liposomes as potential carriers for the oral delivery of insulin. Nanomedicine Nanotechnology, Biol Med. Elsevier Inc. 2014;10:167-76.

54. Huang A, Su Z, Li S, Sun M, Xiao Y, Ping Q, et al. Oral absorption enhancement of salmon calcitonin by using both $\mathrm{N}$-trimethyl chitosan chloride and oligoarginines-modified liposomes as the carriers. Drug Deliv. 2014;21:388-96.

55 Efficacy and safety of liposomal lactoferrin in COVID-19 patients with mild-to-moderate disease and in COVID-19 asymptomatic patients (NCT04475120) [Internet]. Sponsored by University of Rome Tor Vergata; 2020. Available from: https://clinicaltrials. gov/ct2/show/NCT04475120?id=NCT02278822+OR+ NCT04475120\&draw $=2 \&$ rank $=1 \&$ load $=$ cart

56. Chen D, Xia D, Li X, Zhu Q, Yu H, Zhu C, et al. Comparative study of Pluronic ${ }^{\circledR}$ F127-modified liposomes and chitosanmodified liposomes for mucus penetration and oral absorption of cyclosporine A in rats. Int J Pharm. Elsevier B.V. 2013;449:1-9.

57. Chen Y, Lu Y, Chen J, Lai J, Sun J, Hu F, et al. Enhanced bioavailability of the poorly water-soluble drug fenofibrate by using liposomes containing a bile salt. Int $\mathrm{J}$ Pharm. 2009;376:153-60.

58. Jain S, Kumar D, Swarnakar NK, Thanki K. Polyelectrolyte stabilized multilayered liposomes for oral delivery of paclitaxel. Biomaterials Elsevier Ltd. 2012;33:6758-68.

59. Paliwal R, Paliwal SR, Mishra N, Mehta A, Vyas SP. Engineered chylomicron mimicking carrier emulsome for lymph targeted oral delivery of methotrexate. Int J Pharm. 2009;380:181-8.

60. A ğardan NBM, Değim Z, Yilmaz, Altıntaş L, Topal T. Tamoxifen/raloxifene loaded liposomes for oral treatment of breast cancer. J Drug Deliv Sci Technol. 2020;57.

61. Das S, Chaudhury A. Recent advances in lipid nanoparticle formulations with solid matrix for oral drug delivery. AAPS PharmSciTech. 2011;12:62-76. 
62. Salah E, Abouelfetouh MM, Pan Y, Chen D, Xie S. Solid lipid nanoparticles for enhanced oral absorption: A review. Colloids Surfaces B Biointerfaces. Elsevier; 2020;196:111305.

63. Shi LL, Lu J, Cao Y, Liu JY, Zhang XX, Zhang H, et al. Gastrointestinal stability, physicochemical characterization and oral bioavailability of chitosan or its derivative-modified solid lipid nanoparticles loading docetaxel. Drug Dev Ind Pharm. 2017;43:839-46.

64. Pooja D, Kulhari H, Kuncha M, Rachamalla SS, Adams DJ, Bansal V, et al. Improving efficacy, oral bioavailability, and delivery of paclitaxel using protein-grafted solid lipid nanoparticles. Mol Pharm. 2016;13:3903-12.

65. Yuan H, Chen CY, Chai GH, Du YZ, Hu FQ. Improved transport and absorption through gastrointestinal tract by pegylated solid lipid nanoparticles. Mol Pharm. 2013;10:1865-73.

66. Nasirizadeh S, Jaafari MR, Iranshahi M, Golmohammadzadeh S, Mahmoudi A, Ansari L, et al. The effect of efflux pump inhibitors on in vitro and in vivo efficacy of solid lipid nanoparticles containing SN38. J Drug Deliv Sci Technol. Elsevier B.V. 2020;60:101969.

67. Patel MH, Mundada VP, Sawant KK. Fabrication of solid lipid nanoparticles of lurasidone $\mathrm{HCl}$ for oral delivery: optimization, in vitro characterization, cell line studies and in vivo efficacy in schizophrenia. Drug Dev Ind Pharm. Taylor \& Francis 2019;45:1242-57.

68. Patel M, Mundada V, Sawant K. Enhanced intestinal absorption of asenapine maleate by fabricating solid lipid nanoparticles using TPGS: elucidation of transport mechanism, permeability across Caco-2 cell line and in vivo pharmacokinetic studies. Artif Cells, Nanomedicine Biotechnol. Taylor \& Francis 2019;47:144-53.

69. Diwan R, Ravi PR, Pathare NS, Aggarwal V. Pharmacodynamic, pharmacokinetic and physical characterization of cilnidipine loaded solid lipid nanoparticles for oral delivery optimized using the principles of design of experiments. Colloids Surfaces B Biointerfaces. Elsevier 2020;193:111073.

70. El-Say KM, Hosny KM. Optimization of carvedilol solid lipid nanoparticles: an approach to control the release and enhance the oral bioavailability on rabbits. PLoS ONE. 2018;13:1-15.

71. Makwana V, Jain R, Patel K, Nivsarkar M, Joshi A. Solid lipid nanoparticles (SLN) of Efavirenz as lymph targeting drug delivery system: Elucidation of mechanism of uptake using chylomicron flow blocking approach. Int J Pharm. Elsevier B.V. 2015;495:439-46.

72. Dudhipala N, Veerabrahma K. Improved anti-hyperlipidemic activity of Rosuvastatin Calcium via lipid nanoparticles: Pharmacokinetic and pharmacodynamic evaluation. Eur J Pharm Biopharm. Elsevier B.V. 2017;110:47-57.

73. Liu Q, Huang H, Chen H, Lin J, Wang Q. Food-grade nanoemulsions: preparation, stability and application in encapsulation of bioactive compounds. Molecules. 2019;24:1-37.

74. Singh Y, Meher JG, Raval K, Khan FA, Chaurasia M, Jain NK, et al. Nanoemulsion: Concepts, development and applications in drug delivery. J Control Release. Elsevier B.V. 2017;252:28-49.

75. McClements DJ. Nanoemulsions versus microemulsions: terminology, differences, and similarities. Soft Matter. 2012;8:1719-29.

76. McClements DJ. Nanoemulsion-based oral delivery systems for lipophilic bioactive components: nutraceuticals and pharmaceuticals. Ther Deliv. 2013;4:841-57.

77. Sutradhar KB, Amin L. Nanoemulsions: Increasing possibilities in drug delivery. Eur J Nanomedicine. 2013;5:97-110.

78. Anton N, Vandamme TF. Nano-emulsions and microemulsions: clarifications of the critical differences. Pharm Res. 2011;28:978-85.
79. Bouchemal K, Briançon S, Perrier E, Fessi H. Nano-emulsion formulation using spontaneous emulsification: solvent, oil and surfactant optimisation. Int J Pharm. 2004;280:241-51.

80. Saberi AH, Fang Y, McClements DJ. Fabrication of vitamin E-enriched nanoemulsions: Factors affecting particle size using spontaneous emulsification. J Colloid Interface Sci. Elsevier Inc. 2013;391:95-102.

81. Tushar K. Vyas, Aliasgar Shahiwala and MMA. Improved oral bioavailability and brain transport of saquinavir upon administration in novel nanoemulsion formulations. Int J Pharm. 2008;22.

82. Gorain B, Choudhury H, Kundu A, Sarkar L, Karmakar S, Jaisankar P, et al. Nanoemulsion strategy for olmesartan medoxomil improves oral absorption and extended antihypertensive activity in hypertensive rats. Colloids Surfaces B Biointerfaces. Elsevier B.V. 2014;115:286-94.

83. Doh HJ, Jung Y, Balakrishnan P, Cho HJ, Kim DD. A novel lipid nanoemulsion system for improved permeation of granisetron. Colloids Surfaces B Biointerfaces. Elsevier B.V. 2013;101:475-80.

84. Pangeni R, Kang SW, Oak M, Park EY, Park JW. Oral delivery of quercetin in oil-in-water nanoemulsion: in vitro characterization and in vivo anti-obesity efficacy in mice. J Funct Foods Elsevier Ltd. 2017;38:571-81.

85. Vecchione R, Quagliariello V, Calabria D, Calcagno V, De Luca E, Iaffaioli R V, et al. Curcumin bioavailability from oil in water nano-emulsions: In vitro and in vivo study on the dimensional, compositional and interactional dependence. J Control Release. Elsevier B.V. 2016;233:88-100.

86. Hu J, Sun L, Zhao D, Zhang L, Ye M, Tan Q, et al. Supermolecular evodiamine loaded water-in-oil nanoemulsions: Enhanced physicochemical and biological characteristics. Eur J Pharm Biopharm. Elsevier B.V. 2014;88:556-64.

87. Rabelo CAS, Taarji N, Khalid N, Kobayashi I, Nakajima M, Neves MA. Formulation and characterization of water-in-oil nanoemulsions loaded with açaí berry anthocyanins: Insights of degradation kinetics and stability evaluation of anthocyanins and nanoemulsions. Food Res Int Elsevier. 2018;106:542-8.

88. McClements DJ, Rao J. Food-grade nanoemulsions: formulation, fabrication, properties, performance, biological fate, and potential toxicity. Crit Rev Food Sci Nutr. 2011;51:285-330.

89. Tiwari SB, Amiji MM. Improved oral delivery of paclitaxel following administration in nanoemulsion formulations. J Nanosci Nanotechnol. 2006;6:3215-21.

90. Chávez-Zamudio R, Ochoa-Flores AA, Soto-Rodríguez I, Garcia-Varela R, García HS. Preparation, characterization and bioavailability by oral administration of $\mathrm{O} / \mathrm{W}$ curcumin nanoemulsions stabilized with lysophosphatidylcholine. Food Funct. 2017;8:3346-54

91. Desai J, Thakkar H. Enhanced oral bioavailability and brain uptake of Darunavir using lipid nanoemulsion formulation. Colloids Surfaces B Biointerfaces. Elsevier B.V. 2019;175:143-9.

92. Gasa-Falcon A, Odriozola-Serrano I, Oms-Oliu G, Martín-Belloso O. Impact of emulsifier nature and concentration on the stability of $\beta$-carotene enriched nanoemulsions during: In vitro digestion. Food Funct. 2019;10:713-22.

93. Pandey G, Mittapelly N, Valicherla GR, Shukla RP, Sharma S, Banala VT, et al. P-gp modulatory acetyl-11-keto- $\beta$-boswellic acid based nanoemulsified carrier system for augmented oral chemotherapy of docetaxel. Colloids Surfaces B Biointerfaces. Elsevier B.V. 2017;155:276-86.

94. Shahiwala A, Amiji MM. Enhanced mucosal and systemic immune response with squalane oil-containing multiple emulsions upon intranasal and oral administration in mice. J Drug Target. 2008;16:302-10. 
95. Choudhury H, Gorain B, Karmakar S, Biswas E, Dey G, Barik $\mathrm{R}$, et al. Improvement of cellular uptake, in vitro antitumor activity and sustained release profile with increased bioavailability from a nanoemulsion platform. Int J Pharm. Elsevier B.V. 2014;460:131-43.

96. Khandavilli S, Panchagnula R. Nanoemulsions as versatile formulations for paclitaxel delivery: peroral and dermal delivery studies in rats. J Invest Dermatol Elsevier Masson SAS. 2007;127:154-62.

97. Pangeni R, Choi SW, Jeon OC, Byun Y, Park JW. Multiple nanoemulsion system for an oral combinational delivery of oxaliplatin and 5-fluorouracil: preparation and in vivo evaluation. Int J Nanomedicine. 2016;11:6379-99.

98. Choi JU, Maharjan R, Pangeni R, Jha SK, Lee NK, Kweon S, et al. Modulating tumor immunity by metronomic dosing of oxaliplatin incorporated in multiple oral nanoemulsion. J Control Release. 2020;322:13-30.

99. Ge L, He X, Zhang Y, Zhang Y, Chai F, Jiang L, et al. A dabigatran etexilate phospholipid complex nanoemulsion system for further oral bioavailability by reducing drug-leakage in the gastrointestinal tract. Nanomedicine Nanotechnology, Biol Med. Elsevier Inc. 2018;14:1455-64.

100. Gao F, Zhang Z, Bu H, Huang Y, Gao Z, Shen J, et al. Nanoemulsion improves the oral absorption of candesartan cilexetil in rats: Performance and mechanism. J Control Release. Elsevier B.V. 2011;149:168-74.

101. Wan K, Sun L, Hu X, Yan Z, Zhang Y, Zhang X, et al. Novel nanoemulsion based lipid nanosystems for favorable in vitro and in vivo characteristics of curcumin. Int J Pharm. Elsevier B.V. 2016;504:80-8.

102. Young NA, Bruss MS, Gardner M, Willis WL, Mo X, Valiente $\mathrm{GR}$, et al. Oral administration of nano-emulsion curcumin in mice suppresses inflammatory-induced NFkB signaling and macrophage migration. PLoS One. 2014;9.

103. Artiga-Artigas M, Lanjari-Pérez Y, Martín-Belloso O. Curcuminloaded nanoemulsions stability as affected by the nature and concentration of surfactant. Food Chem Elsevier. 2018;266:466-74.

104. Salvia-Trujillo L, Verkempinck SHE, Zhang X, Van Loey AM, Grauwet T, Hendrickx ME. Comparative study on lipid digestion and carotenoid bioaccessibility of emulsions, nanoemulsions and vegetable-based in situ emulsions. Food Hydrocoll. Elsevier B.V. 2019;87:119-28.

105. Yao K, McClements DJ, Xiang J, Zhang Z, Cao Y, Xiao H, et al. Improvement of carotenoid bioaccessibility from spinach by co-ingesting with excipient nanoemulsions: impact of the oil phase composition. Food Funct. 2019;10:5302-11.

106. Sessa M, Balestrieri ML, Ferrari G, Servillo L, Castaldo D, D'Onofrio N, et al. Bioavailability of encapsulated resveratrol into nanoemulsion-based delivery systems. Food Chem Elsevier Ltd. 2014;147:42-50.

107. Guo RX, Fu X, Chen J, Zhou L, Chen G. Preparation and characterization of microemulsions of myricetin for improving its antiproliferative and antioxidative activities and oral bioavailability. J Agric Food Chem. 2016;64:6286-94.

108. Langella A, Calcagno V, De Gregorio V, Urciuolo F, Imparato $\mathrm{G}$, Vecchione $\mathrm{R}$, et al. In vitro study of intestinal epithelial interaction with engineered oil in water nanoemulsions conveying curcumin. Colloids Surfaces B Biointerfaces. Elsevier B.V. 2018;164:232-9.

109. Pinheiro AC, Coimbra MA, Vicente AA. In vitro behaviour of curcumin nanoemulsions stabilized by biopolymer emulsifiers effect of interfacial composition. Food Hydrocoll. 2016;52:460-7.

110. Silva HD, Poejo J, Pinheiro AC, Donsì F, Serra AT, Duarte CMM, et al. Evaluating the behaviour of curcumin nanoemulsions and multilayer nanoemulsions during dynamic in vitro digestion. $\mathbf{J}$ Funct Foods Elsevier. 2018;48:605-13.
111 Pilot study of curcumin for women with obesity and high risk for breast cancer (NCT01975363) [Internet]. Sponsored by Ohio State University Comprehensive Cancer Center; 2013. Available from: https://clinicaltrials.gov/ct2/show/NCT01975363

112 Curcumin in reducing joint pain in breast cancer survivors with aromatase inhibitor-induced joint disease (NCT03865992) [Internet]. Sponsored by city of Hope Medical Center; 2020. Available from: https://clinicaltrials.gov/ct2/show/NCT03865992

113. Date AA, Desai N, Dixit R, Nagarsenker M. Self-nanoemulsifying drug delivery systems: formulation insights, applications and advances. Nanomedicine. 2010;5:1595-616.

114. Guo Y, Mao X, Zhang J, Sun P, Wang H, Zhang Y, et al. Oral delivery of lycopene-loaded microemulsion for braintargeting: preparation, characterization, pharmacokinetic evaluation and tissue distribution. Drug Deliv Taylor \& Francis. 2019;26:1191-205.

115. Xiao Y, Chen X, Yang L, Zhu X, Zou L, Meng F, et al. Preparation and oral bioavailability study of curcuminoid-loaded microemulsion. J Agric Food Chem. 2013;61:3654-60.

116. Hirunpanich V, Sato H. Improvement of cyclosporine A bioavailability by incorporating ethyl docosahexaenoate in the microemulsion as an oil excipient. Eur J Pharm Biopharm. Elsevier B.V. 2009;73:247-52.

117. Black KCL, Wang Y, Luehmann HP, Cai X, Xing W, Pang B, et al. Radioactive 198Au-doped nanostructures with different shapes for in vivo analyses of their biodistribution, tumor uptake, and intratumoral distribution. ACS Nano. 2014;8:4385-94.

118. Agarwal R, Jurney P, Raythatha M, Singh V, Sreenivasan SV, Shi L, et al. Effect of shape, size, and aspect ratio on nanoparticle penetration and distribution inside solid tissues using 3D spheroid models. Adv Healthc Mater. 2015;4:2269-80.

119. Decuzzi P, Ferrari M. The receptor-mediated endocytosis of nonspherical particles. Biophys J Elsevier. 2008;94:3790-7.

120. Yang K, Ma YQ. Computer simulation of the translocation of nanoparticles with different shapes across a lipid bilayer. Nat Nanotechnol Nature Publishing Group. 2010;5:579-83.

121. Sang KK, Eun HL, Vaishali B, Lee S, Lee YK, Kim CY, et al. Tricaprylin microemulsion for oral delivery of low molecular weight heparin conjugates. J Control Release. 2005;105:32-42.

122. Li Y, Yokoyama W, Xu S, Zhu S, Ma J, Zhong F. Formation and stability of W/O microemulsion formed by food grade ingredients and its oral delivery of insulin in mice. J Funct Foods Elsevier Ltd. 2017;30:134-41.

123. Yin YM, Cui F De, Mu CF, Choi MK, Kim JS, Chung SJ, et al. Docetaxel microemulsion for enhanced oral bioavailability: Preparation and in vitro and in vivo evaluation. J Control Release. Elsevier B.V. 2009;140:86-94.

124. Lambert G, Fattal E, Pinto-Alphandary H, Gulik A, Couvreur P. Polyisobutylcyanoacrylate nanocapsules containing an aqueous core for the delivery of oligonucleotides. Int J Pharm. 2001;214:13-6.

125. Hillaireau H, Le Doan T, Besnard M, Chacun H, Janin J, Couvreur P. Encapsulation of antiviral nucleotide analogues azidothymidine-triphosphate and cidofovir in poly(isobutylcyanoacrylate) nanocapsules. Int J Pharm. 2006;324:37-42.

126. Calvo $\mathrm{P}$, Remuñán-López $\mathrm{C}$, Vila-Jato JL, Alonso MJ. Development of positively charged colloidal drug carriers: chitosan-coated polyester nanocapsules and submicron-emulsions. Colloid Polym Sci. 1997;275:46-53.

127. Ramadan A, Lagarce F, Tessier-Marteau A, Thomas O, Legras $\mathrm{P}$, Macchi L, et al. Oral fondaparinux: use of lipid nanocapsules as nanocarriers and in vivo pharmacokinetic study. Int $\mathbf{J}$ Nanomedicine. 2011;6:2941-51.

128. Roger E, Lagarce F, Benoit JP. Development and characterization of a novel lipid nanocapsule formulation of Sn38 for oral administration. Eur J Pharm Biopharm. Elsevier B.V. 2011;79:181-8. 
129. Groo AC, Saulnier P, Gimel JC, Gravier J, Ailhas C, Benoit JP, et al. Fate of paclitaxel lipid nanocapsules in intestinal mucus in view of their oral delivery. Int J Nanomedicine. 2013;8:4291-302.

130. Peltier S, Oger JM, Lagarce F, Couet W, Benoît JP. Enhanced oral paclitaxel bioavailability after administration of paclitaxel-loaded lipid nanocapsules. Pharm Res. 2006;23:1243-50.

131. Xu Y, Van Hul M, Suriano F, Préat V, Cani PD, Beloqui A. Novel strategy for oral peptide delivery in incretin-based diabetes treatment. Gut. 2020;69:911-9.

132. Friedrich RB, Dimer FA, Guterres SS, Beck RCR, Pohlmann AR. Nanoencapsulation of tacrolimus in lipid-core nanocapsules showed similar immunosuppressive activity after oral and intraperitoneal administrations. J Biomed Nanotechnol. 2014;10:1599-609.

133. Yin J, Xiang C, Song X. Nanoencapsulation of psoralidin via chitosan and Eudragit S100 for enhancement of oral bioavailability. Int J Pharm. Elsevier B.V. 2016;510:203-9.

134. Michalowski CB, Arbo MD, Altknecht L, Anciuti AN, Abreu ASG, Alencar LMR, et al. Oral treatment of spontaneously hypertensive rats with captopril-surface functionalized furosemide-loaded multi-wall lipid-core nanocapsules. Pharmaceutics. 2020;12.

135. Bapat P, Ghadi R, Chaudhari D, Katiyar SS, Jain S. Tocophersolan stabilized lipid nanocapsules with high drug loading to improve the permeability and oral bioavailability of curcumin. Int J Pharm Elsevier. 2019;560:219-27.

136. Ranganathan A, Hindupur R, Vallikannan B. Biocompatible lutein-polymer-lipid nanocapsules: Acute and subacute toxicity and bioavailability in mice. Mater Sci Eng C. Elsevier B.V. 2016;69:1318-27.

137. de Oliveira MTP, Coutinho D de S, de Souza ÉT, Guterres SS, Pohlmann AR, Silva PMR, et al. Orally delivered resveratrolloaded lipid-core nanocapsules ameliorate LPS-induced acute lung injury via the ERK and PI3K/Akt pathways. Int J Nanomedicine. 2019;14:5215-28.

138. Frozza RL, Bernardi A, Paese K, Hoppe JB, Da Silva T, Battastini AMO, et al. Characterization of trans-resveratrolloaded lipid-core nanocapsules and tissue distribution studies in rats. J Biomed Nanotechnol. 2010;6:694-703.

139. Pouton CW. Self-emulsifying drug delivery systems: assessment of the efficiency of emulsification. Int J Pharm. 1985;27:335-48.

140. Khan AW, Kotta S, Ansari SH, Sharma RK, Ali J. Potentials and challenges in self-nanoemulsifying drug delivery systems. Expert Opin Drug Deliv. 2012;9:1305-17.

141. Dokania S, Joshi AK. Self-microemulsifying drug delivery system (SMEDDS)-challenges and road ahead. Drug Deliv. 2015;22:675-90.

142. Reiss H. Entropy-induced dispersion of bulk liquids. J Colloid Interface Sci. 1975;53:61-70.

143. Karwal R, Garg T, Rath G, Markandeywar TS. Current trends in self-emulsifying drug- delivery systems (SEDDSs) to enhance the bioavailability of poorly water-soluble drugs. Crit Rev Ther Drug Carrier Syst. 2016;33:1-39.

144. Singh G, Pai RS. Optimized self-nanoemulsifying drug delivery system of atazanavir with enhanced oral bioavailability: in vitro/in vivo characterization. Expert Opin Drug Deliv. 2014;11:1023-32.

145. Janković J, Djekic L, Dobričić V, Primorac M. Evaluation of critical formulation parameters in design and differentiation of self-microemulsifying drug delivery systems (SMEDDSs) for oral delivery of aciclovir. Int J Pharm. 2016;497:301-11.

146. Basalious EB, Shawky N, Badr-Eldin SM. SNEDDS containing bioenhancers for improvement of dissolution and oral absorption of lacidipine. I: Development and optimization. Int J Pharm. Elsevier B.V. 2010;391:203-11.
147. AboulFotouh K, Allam AA, El-Badry M, El-Sayed AM. A Self-nanoemulsifying drug delivery system for enhancing the oral bioavailability of candesartan cilexetil: ex vivo and in vivo evaluation. J Pharm Sci. 2019;108:3599-608.

148. Beg S, Sharma G, Thanki K, Jain S, Katare OP, Singh B. Positively charged self-nanoemulsifying oily formulations of olmesartan medoxomil: Systematic development, in vitro, ex vivo and in vivo evaluation. Int J Pharm. Elsevier B.V. 2015;493:466-82.

149. Goo YT, Song SH, Yeom DW, Chae BR, Yoon HY, Kim CH, et al. Enhanced oral bioavailability of valsartan in rats using a supersaturable self-microemulsifying drug delivery system with P-glycoprotein inhibitors. Pharm Dev Technol Taylor \& Francis. 2020;25:178-86.

150. Han H, Li Y, Peng Z, Long K, Zheng C, Wang W, et al. A Soluplus/Poloxamer 407-based self-nanoemulsifying drug delivery system for the weakly basic drug carvedilol to improve its bioavailability. Nanomedicine Nanotechnology, Biol Med. Elsevier Inc. 2020;27:102199.

151. Akhtar N, Talegaonkar S, Khar RK, Jaggi M. Selfnanoemulsifying lipid carrier system for enhancement of oral bioavailability of etoposide by P-glycoprotein modulation: In vitro cell line and in vivo pharmacokinetic investigation. J Biomed Nanotechnol. 2013;9:1216-29.

152. Cui W, Zhao H, Wang C, Chen Y, Luo C, Zhang S, et al. Co-encapsulation of docetaxel and cyclosporin A into SNEDDS to promote oral cancer chemotherapy. Drug Deliv Taylor \& Francis. 2019;26:542-50.

153. Jain AK, Thanki K, Jain S. Solidified self-nanoemulsifying formulation for oral delivery of combinatorial therapeutic regimen: Part II in vivo pharmacokinetics, antitumor efficacy and hepatotoxicity. Pharm Res. 2014;31:946-58.

154. Seo YG, Kim DH, Ramasamy T, Kim JH, Marasini N, Oh YK, et al. Development of docetaxel-loaded solid self-nanoemulsifying drug delivery system (SNEDDS) for enhanced chemotherapeutic effect. Int J Pharm. Elsevier B.V. 2013;452:412-20.

155. Sandhu PS, Beg S, Mehta F, Singh B, Trivedi P. Novel dietary lipid-based self-nanoemulsifying drug delivery systems of paclitaxel with p-gp inhibitor: implications on cytotoxicity and biopharmaceutical performance. Expert Opin Drug Deliv. 2015;12:1809-22.

156. Benival DM, Devarajan PV. In situ lipidization as a new approach for the design of a self microemulsifying drug delivery system (SMEDDS) of doxorubicin hydrochloride for oral administration. J Biomed Nanotechnol. 2015;11:913-22.

157. Kim DS, Cho JH, Park JH, Kim JS, Song ES, Kwon J, et al. Self-microemulsifying drug delivery system (SMEDDS) for improved oral delivery and photostability of methotrexate. Int $\mathbf{J}$ Nanomedicine. 2019;14:4949-60.

158. Xie M, Wu J, Ji L, Jiang X, Zhang J, Ge M, et al. Development of triptolide self-microemulsifying drug delivery system and its anti-tumor effect on gastric cancer xenografts. Front Oncol. 2019;9:1-10.

159. Beg S, Jena SS, Patra CN, Rizwan M, Swain S, Sruti J, et al. Development of solid self-nanoemulsifying granules (SSNEGs) of ondansetron hydrochloride with enhanced bioavailability potential. Colloids Surfaces B Biointerfaces. Elsevier B.V. 2013;101:414-23.

160. Wang Y, Sun J, Zhang T, Liu H, He F, He Z. Enhanced oral bioavailability of tacrolimus in rats by self- microemulsifying drug delivery systems. Drug Dev Ind Pharm. 2011;37:1225-30.

161. Li P, Tan A, Prestidge CA, Nielsen HM, Müllertz A. Selfnanoemulsifying drug delivery systems for oral insulin delivery: In vitro and in vivo evaluations of enteric coating and drug loading. Int J Pharm. Elsevier B.V. 2014;477:390-8.

162. Li P, Nielsen HM, Müllertz A. Impact of lipid-based drug delivery systems on the transport and uptake of insulin across Caco-2 cell monolayers. J Pharm Sci Elsevier Ltd. 2016;105:2743-51. 
163. Zhang Q, He N, Zhang L, Zhu F, Chen Q, Qin Y, et al. The in vitro and in vivo study on Self-Nanoemulsifying Drug Delivery System (SNEDDS) based on insulin-phospholipid complex. J Biomed Nanotechnol. 2012;8:90-7.

164. Sakloetsakun D, Dünnhaupt S, Barthelmes J, Perera G, BernkopSchnürch A. Combining two technologies: Multifunctional polymers and self-nanoemulsifying drug delivery system (SNEDDS) for oral insulin administration. Int J Biol Macromol. Elsevier B.V. 2013;61:363-72.

165. Qi X, Wang L, Zhu J, Hu Z, Zhang J. Self-double-emulsifying drug delivery system (SDEDDS): A new way for oral delivery of drugs with high solubility and low permeability. Int J Pharm. Elsevier B.V. 2011;409:245-51.

166. Taha EI, Al-Suwayeh SA, Anwer MK. Preparation, in vitro and in vivo evaluation of solid-state self-nanoemulsifying drug delivery system (SNEDDS) of vitamin A acetate. J Drug Target. 2009; 17:468-73.

167. Sangsen Y, Wiwattanawongsa K, Likhitwitayawuid K, Sritularak B, Graidist P, Wiwattanapatapee R. Influence of surfactants in self-microemulsifying formulations on enhancing oral bioavailability of oxyresveratrol: Studies in Caco-2 cells and in vivo. Int J Pharm. Elsevier B.V. 2016;498:294-303.

168. Mamadou G, Charrueau C, Dairou J, Limas Nzouzi N, Eto B, Ponchel G. Increased intestinal permeation and modulation of presystemic metabolism of resveratrol formulated into selfemulsifying drug delivery systems. Int J Pharm. Elsevier B.V. 2017;521:150-5.

169. Qian J, Meng H, Xin L, Xia M, Shen H, Li G, et al. Selfnanoemulsifying drug delivery systems of myricetin: Formulation development, characterization, and in vitro and in vivo evaluation. Colloids Surfaces B Biointerfaces. Elsevier B.V. 2017;160:101-9.

170. Chou YC, Li S, Ho CT, Pan MH. Preparation and evaluation of self-microemulsifying delivery system containing 5-demethyltangeretin on inhibiting xenograft tumor growth in mice. Int J Pharm. Elsevier B.V. 2020;579:119134.

171. Cui J, Yu B, Zhao Y, Zhu W, Li H, Lou H, et al. Enhancement of oral absorption of curcumin by self-microemulsifying drug delivery systems. Int J Pharm. 2009;371:148-55.

172. Dou YX, Zhou JT, Wang TT, Huang YF, Chen VP, Xie YL, et al. Self-nanoemulsifying drug delivery system of bruceine D: a new approach for anti-ulcerative colitis. Int J Nanomedicine. 2018;13:5887-907.

173. Liao H, Gao Y, Lian C, Zhang Y, Wang B, Yang Y, et al. Oral absorption and lymphatic transport of baicalein following drugphospholipid complex incorporation in self-microemulsifying drug delivery systems. Int J Nanomedicine. 2019;14:7291-306.

174. Hauer B, Meinzer A, Posanski U, Ritcher F. United States Patent ( 19 ). United States 1994.

175. Rosenberg, Joerg E, Reinhold, Ulrich H, Liepold, Bernd D, Berndl, Gunther, Herxheim M. ( 12 ) United States Patent. United States 2012.

176. Lei B, Zha W, Wang Y, Wen C, Stude EJ, Wang X, et al. NIH Public Access. 2011;7:844-53.

177. Verwey EJW, Overbeek JTG. Theory of the stability of lyophobic colloids. J. Colloid Sci. 1955. p. 224-5.

178. Derjaguin B, Landau L. Theory of the stability of strongly charged lyophobic sols and of the adhesion of strongly charged particles in solutions of electrolytes. Prog Surf Sci. 1993;43:30-59.

179. Olbrich C, Mu RH, Berlin D. Enzymatic degradation of SLNeffect of surfactant and pdf. Int J Pharm. 1999;180:31-9.

180. Chen C, Fan T, Jin Y, Zhou Z, Yang Y, Zhu X, et al. Orally delivered salmon calcitonin-loaded solid lipid nanoparticles prepared by micelle-double emulsion method via the combined use of different solid lipids. Nanomedicine. 2013;8:1085-100.
181. Kashanian S, Rostami E. PEG-stearate coated solid lipid nanoparticles as levothyroxine carriers for oral administration. J Nanoparticle Res. 2014;16.

182. Garcia-Fuentes M, Torres D, Alonso MJ. New surface-modified lipid nanoparticles as delivery vehicles for salmon calcitonin. Int J Pharm. 2005;296:122-32.

183. Santander-Ortega MJ, Peula-García JM, Goycoolea FM, OrtegaVinuesa JL. Chitosan nanocapsules: Effect of chitosan molecular weight and acetylation degree on electrokinetic behaviour and colloidal stability. Colloids Surfaces B Biointerfaces. Elsevier B.V. 2011;82:571-80.

184. Wu W, Niu M, Lu Y, Hovgaard L. Liposomes containing glycocholate as potential oral insulin delivery systems: preparation, in vitro characterization, and improved protection against enzymatic degradation. Int J Nanomedicine. 2011;1155.

185. Hu S, Niu M, Hu F, Lu Y, Qi J, Yin Z, et al. Integrity and stability of oral liposomes containing bile salts studied in simulated and ex vivo gastrointestinal media. Int J Pharm. 2013;441:693-700.

186. Liu Q, Chen J, Qin Y, Jiang B, Zhang T. Encapsulation of pterostilbene in nanoemulsions: influence of lipid composition on physical stability, in vitro digestion, bioaccessibility, and Caco-2 cell monolayer permeability. Food Funct. 2019;10:6604-14.

187. Plaza-Oliver M, Baranda JFSD, Rodríguez Robledo V, CastroVázquez L, Gonzalez-Fuentes J, Marcos P, et al. Design of the interface of edible nanoemulsions to modulate the bioaccessibility of neuroprotective antioxidants. Int J Pharm. 2015;490.

188. Müller C, Leithner K, Hauptstein S, Hintzen F, Salvenmoser W, Bernkop-Schnürch A. Preparation and characterization of mucuspenetrating papain/poly(acrylic acid) nanoparticles for oral drug delivery applications. J Nanoparticle Res. 2013;15.

189. Zhang S, Asghar S, Yu F, Hu Z, Ping Q, Chen Z, et al. The enhancement of $\mathrm{N}$-acetylcysteine on intestinal absorption and oral bioavailability of hydrophobic curcumin. Eur J Pharm Sci. Elsevier B.V. 2020;105506.

190. MacAdam A. The effect of gastro-intestinal mucus on drug absorption. Adv Drug Deliv Rev. 1993;11:201-20.

191. Lai SK, Wang YY, Hanes J. Mucus-penetrating nanoparticles for drug and gene delivery to mucosal tissues. Adv Drug Deliv Rev. Elsevier B.V. 2009;61:158-71.

192. Federer C, Kurpiers M, Bernkop-Schnürch A. Thiolated chitosans: a multi-talented class of polymers for various applications. Biomacromolecules. 2020.

193. Silva HD, Beldíková E, Poejo J, Abrunhosa L, Serra AT, Duarte CMM, et al. Evaluating the effect of chitosan layer on bioaccessibility and cellular uptake of curcumin nanoemulsions. J Food Eng. 2019;243:89-100.

194. Prego C, Fabre M, Torres D, Alonso MJ. Efficacy and mechanism of action of chitosan nanocapsules for oral peptide delivery. Pharm Res. 2006;23:549-56.

195. Sánchez-Moreno P, Ortega-Vinuesa JL, Martín-Rodríguez A, Boulaiz H, Marchal-Corrales JA, Peula-García JM. Characterization of different functionalized lipidic nanocapsules as potential drug carriers. Int J Mol Sci. 2012;13:2405-24.

196. Santander-Ortega MJ, Lozano-López MV, Bastos-González D, Peula-García JM, Ortega-Vinuesa JL. Novel core-shell lipidchitosan and lipid-poloxamer nanocapsules: stability by hydration forces. Colloid Polym Sci. 2010;288:159-72.

197. Prego C, García M, Torres D, Alonso MJ. Transmucosal macromolecular drug delivery. J Control Release. 2005;101:151-62.

198. Gradauer K, Vonach C, Leitinger G, Kolb D, Fröhlich E, Roblegg E, et al. Chemical coupling of thiolated chitosan to preformed liposomes improves mucoadhesive properties. Int $\mathrm{J}$ Nanomedicine. 2012;7:2523-34.

199. Li X, Qi J, Xie Y, Zhang X, Hu S, Xu Y, et al. Nanoemulsions coated with alginate/chitosan as oral insulin delivery systems: 
Preparation, characterization, and hypoglycemic effect in rats. Int J Nanomedicine. 2012;8:23-32.

200. Cattani VB, Fiel LA, Jäger A, Jäger E, Colomé LM, Uchoa F, et al. Lipid-core nanocapsules restrained the indomethacin ethyl ester hydrolysis in the gastrointestinal lumen and wall acting as mucoadhesive reservoirs. Eur J Pharm Sci. 2010;39:116-24.

201. Adamczak MI, Hagesaether E, Smistad G, Hiorth M. An in vitro study of mucoadhesion and biocompatibility of polymer coated liposomes on HT29-MTX mucus-producing cells. Int J Pharm. 2016;498:225-33.

202. Thirawong N, Thongborisute J, Takeuchi H, Sriamornsak P. Improved intestinal absorption of calcitonin by mucoadhesive delivery of novel pectin-liposome nanocomplexes. J Control Release. 2008;125:236-45.

203. Sakloetsakun D, Perera G, Hombach J, Millotti G, BernkopSchnürch A. The impact of vehicles on the mucoadhesive properties of orally administrated nanoparticles: a case study with chitosan-4-thiobutylamidine conjugate. AAPS PharmSciTech. 2010;11:1185-92.

204. Lai SK, O'Hanlon DE, Harrold S, Man ST, Wang YY, Cone $\mathrm{R}$, et al. Rapid transport of large polymeric nanoparticles in fresh undiluted human mucus. Proc Natl Acad Sci U S A. 2007; 104:1482-7.

205. Wang YY, Lai SK, Suk JS, Pace A, Cone R, Hanes J. Addressing the PEG mucoadhesivity paradox to engineer nanoparticles that "slip" through the human mucus barrier. Angew Chemie - Int Ed. 2008;47:9726-9.

206. Huckaby JT, Lai SK. PEGylation for enhancing nanoparticle diffusion in mucus. Adv Drug Deliv Rev. Elsevier B.V. 2018;124:125-39.

207. Li X, Chen D, Le C, Zhu C, Gan Y, Hovgaard L, et al. Novel mucus-penetrating liposomes as a potential oral drug delivery system: preparation, in vitro characterization, and enhanced cellular uptake. Int J Nanomedicine. 2011;6:3151-62.

208. Liu Y, Yang T, Wei S, Zhou C, Lan Y, Cao A, et al. Mucus adhesion- and penetration-enhanced liposomes for paclitaxel oral delivery. Int J Pharm Elsevier. 2018;537:245-56.

209. Abdulkarim M, Agulló N, Cattoz B, Griffiths P, BernkopSchnürch A, Gómez Borros S, et al. Nanoparticle diffusion within intestinal mucus: Three-dimensional response analysis dissecting the impact of particle surface charge, size and heterogeneity across polyelectrolyte, pegylated and viral particles. Eur J Pharm Biopharm. Elsevier B.V. 2015;97:230-8.

210. Berardi A, Bombelli FB, Berardi A. Oral delivery of nanoparticles - let' s not forget about the protein corona. Expert Opin Drug Deliv. Taylor \& Francis 2019;16:563-6.

211. Akhtar N, Ahad A, Khar RK, Jaggi M, Aqil M, Iqbal Z, et al. The emerging role of P-glycoprotein inhibitors in drug delivery: a patent review. Expert Opin Ther Pat. 2011;21:561-76.

212. Yen CC, Chen YC, Wu MT, Wang CC, Wu YT. Nanoemulsion as a strategy for improving the oral bioavailability and antiinflammatory activity of andrographolide. Int J Nanomedicine. 2018;13:669-80.

213. Zhou K, Yan Y, Chen D, Huang L, Li C, Meng K, et al. Solid lipid nanoparticles for duodenum targeted oral delivery of tilmicosin. Pharmaceutics. 2020;12:1-19.
214. Xu Y, Shrestha N, Préat V, Beloqui A. Overcoming the intestinal barrier: a look into targeting approaches for improved oral drug delivery systems. J Control Release. 2020;322:486-508.

215. Ling SSN, Yuen KH, Magosso E, Barker SA. Oral bioavailability enhancement of a hydrophilic drug delivered via folic acidcoupled liposomes in rats. J Pharm Pharmacol. 2009;61:445-9.

216. Zhang N, Ping Q, Huang G, Xu W, Cheng Y, Han X. Lectinmodified solid lipid nanoparticles as carriers for oral administration of insulin. Int J Pharm. 2006;327:153-9.

217. Kang SK, Woo JH, Kim MK, Woo SS, Choi JH, Lee HG, et al. Identification of a peptide sequence that improves transport of macromolecules across the intestinal mucosal barrier targeting goblet cells. J Biotechnol. 2008;135:210-6.

218. Tian Q, Ding F, Guo L, Wang J, Wu F, Yu Y. Targeted solid lipid nanoparticles with peptide ligand for oral delivery of atorvastatin calcium. RSC Adv Royal Society of Chemistry. 2016;6:35901-9.

219. Gupta PN, Vyas SP. Investigation of lectinized liposomes as $\mathrm{M}$-cell targeted carrier-adjuvant for mucosal immunization. Colloids Surfaces B Biointerfaces. Elsevier B.V. 2011;82:118-25.

220. Shrestha N, Bouttefeux O, Vanvarenberg K, Lundquist P, Cunarro $\mathrm{J}$, Tovar S, et al. The stimulation of GLP-1 secretion and delivery of GLP-1 agonists: via nanostructured lipid carriers. Nanoscale Royal Society of Chemistry. 2018;10:603-13.

221. Xu Y, Carradori D, Alhouayek M, Muccioli GG, Cani PD, Préat V, et al. Size effect on lipid nanocapsule-mediated GLP-1 secretion from enteroendocrine L cells. Mol Pharm. 2018;15:108-15.

222. Trevaskis NL, Charman WN, Porter CJH. Lipid-based delivery systems and intestinal lymphatic drug transport: a mechanistic update. Adv Drug Deliv Rev. 2008;60:702-16.

223. Porter CJH, Charman WN. Intestinal lymphatic drug transport: an update. Adv Drug Deliv Rev. 2001;50:61-80.

224. Ge W, Hu PZ, Huang Y, Wang XM, Zhang XM, Sun YJ, et al. The antitumor immune responses induced by nanoemulsionencapsulated MAGE1-HSP70/SEA complex protein vaccine following different administration routes. Oncol Rep. 2009;22:915-20.

225. Garg B, Katare OP, Beg S, Lohan S, Singh B. Systematic development of solid self-nanoemulsifying oily formulations (S-SNEOFs) for enhancing the oral bioavailability and intestinal lymphatic uptake of lopinavir. Colloids Surfaces B Biointerfaces. Elsevier B.V. 2016;141:611-22.

226. Bala V, Rao S, Bateman E, Keefe D, Wang S, Prestidge CA. Enabling oral SN38-based chemotherapy with a combined lipophilic prodrug and self-microemulsifying drug delivery system. Mol Pharm. 2016;13:3518-25.

227. Pokale R, Bandivadekar M. Self micro-emulsifying drug delivery system for lymphatic uptake of darunavir. J Drug Discov Dev Deliv. 2016;3:1-7.

228. O'Driscoll CM. Lipid-based formulations for intestinal lymphatic delivery. Eur J Pharm Sci. 2002;15:405-15.

Publisher's Note Springer Nature remains neutral with regard to jurisdictional claims in published maps and institutional affiliations. 